\title{
Resilience in a Natural Model of Metabolic Dysfunction Through Changes in Longevity and Ageing-Related Metabolites
}

\author{
J Kyle Medley ${ }^{1, \dagger, \Phi}$, Jenna Persons ${ }^{1,2, \dagger}$, Tathagata Biswas ${ }^{1}$, Luke Olsen $^{1,3}$, Robert Peuß $^{1,4}$, Jaya Krishnan ${ }^{1}$, Shaolei Xiong ${ }^{1}$, \\ and Nicolas Rohner ${ }^{1,3,0]}$ \\ ${ }^{1}$ Stowers Institute for Medical Research, $1000 \mathrm{E} 50^{\text {th }}$ Street, Kansas City, Missouri, U.S.A. \\ ${ }^{2}$ Current Address: Newman University, 3100 McCormick St, Wichita, Kansas, U.S.A. \\ ${ }^{3}$ Department of Molecular and Integrative Physiology, University of Kansas Medical Center, 3901 Rainbow Blvd, Kansas City, Kansas, U.S.A. \\ ${ }^{4}$ Current Address: University of Münster, Schlossplatz 2, 48149 Münster, Germany \\ ${ }^{\dagger}$ These authors contributed equally to this work.
}

\begin{abstract}
Insights from extreme-adapted organisms, which have evolved natural strategies for promoting survivability under severe environmental pressures, may help guide future research into novel approaches for enhancing human longevity. The cave-adapted Mexican tetra, Astyanax mexicanus, has attracted interest as a model system for metabolic resilience, a term we use to denote the property of maintaining robust health and longevity under conditions that would have highly deleterious effects in other organisms (Fig 1). Cave-dwelling populations of Mexican tetra exhibit elevated blood glucose and possess a mutation in the insulin receptor that in humans has been linked to Rabson-Mendenhall syndrome, a condition characterized by severe insulin resistance that causes numerous developmental abnormalities, is highly associated with debilitating progression, and drastically reduces lifespan. In addition, cavefish develop large numbers of hypertrophic visceral adipocytes and possess vastly enriched stores of body fat compared to surface-dwelling counterparts. However, cavefish appear to avoid the progression of the respective pathologies typically associated with these conditions, such as accumulation of advanced glycation end products (AGEs), chronic tissue inflammation, impaired growth due to insulin dysregulation, and low survivability due to arterial disease. The metabolic strategies underlying the resilience properties of A. mexicanus cavefish, and how they relate to environmental challenges of the cave environment, are poorly understood. Here, we provide an untargeted metabolomics study of long- and short-term fasting in two $A$. mexicanus cave populations and one surface population. We find that, although cave-
\end{abstract}

Fig. 1. Metabolic resilience - survivability under a variety of extreme conditions. Certain populations of cavefish have adaptations that cause increased appetite (1) and increased fat accumulation (2) (in cases where nutrients are plentiful, such as in lab-raised populations). These same populations also exhibit robust health and longevity $(2,3)$ and do not suffer ill-effects due to high levels of visceral fat and hyperglycemia, both of which are features of most cave populations. However, visceral fat accumulation in cave populations is highly dependent on nutrient availability and is not
displayed in wild-caught specimens (4). Thus, cavefish paradoxically appear to tolerdisplayed in wild-caught specimens (4). Thus, cavefish paradoxically appear to toler-
ate both extremely low and extremely high levels of triglycerides, glucose, and other energy storage metabolites. We argue that these differences can be reconciled under a hypothesis whereby the cave environment selects not for resistance to nutrient deprivation per se, but rather resilience to a variety of nutrient availability states (such as seasonal floods). Survival under such challenging conditions ostensibly favors the ability to tolerate extreme metabolic states, including not only starvation but also high levels of potentially deleterious metabolites such as triglycerides and reactive oxygen
species (ROS). We find evidence for elevated antioxidant levels and altered cholesterol / cholesteryl ester homeostasis in cavefish, suggesting that cavefish may use these
mechanisms to offset potentially harmful metabolites and tolerate a broad range of metabolic conditions. fish share many similarities with metabolic syndrome normally associated with the human state of obesity, important differences emerge, including a reduction in cholesteryl esters and intermediates of protein glycation, and an increase in antioxidants and metabolites associated with hypoxia and longevity. We find important overlaps between metabolic alterations in cave-dwelling Mexican tetra and other models of resilience and extreme longevity, such as naked mole-rats, including enhanced reliance on sugars as an energy source and a trend toward more potent antioxidant activity. This work suggests that certain metabolic features associated with human pathologies are not intrinsically harmful, but are rather consequences of suboptimal adaptation of humans to survival under adverse metabolic conditions, and suggests promising avenues for future investigation into the role of metabolic strategies in evolutionary adaptation and health. We provide a transparent pipeline for reproducing our analysis and a Shiny app for other researchers to explore and visualize our dataset.

Metabolic syndrome | Obesity | Metabolomics | Extreme adaptation | Evolution

Correspondence: nro@stowers.org (Nicolas Rohner), kyle@how2cell.com ( $J$ Kyle Medley)

\section{Introduction}

Metabolism plays a central role in many cellular processes, and its dysregulation is a hallmark of many disease states, including cancer, obesity, and diabetes. Recent work (5) has

\section{Surface Cave environment environment}

-Numerous predators

at all life stages

- Abundant and

diverse pathogens

Plentiful food

$\bullet$ High maximum

population density

Selection favors

rapid growth and

intergenerational

gap, and high-risk

energy allocation.

Metabolic pathways wired to exploit available resources protection from metabolic stress and harmful metabolic byproducts less of a concern.

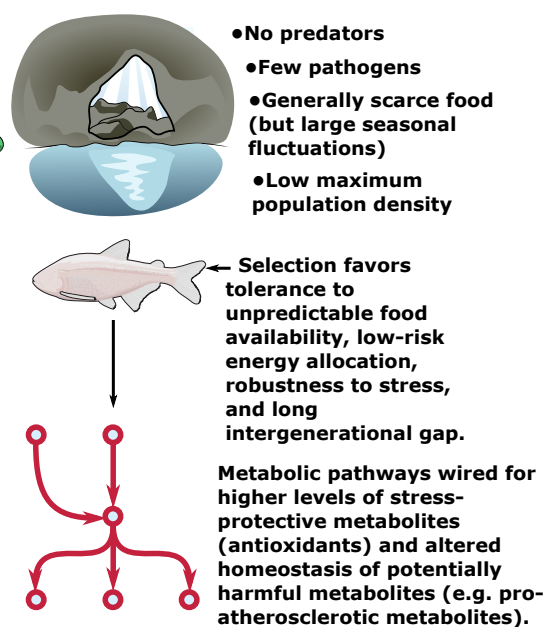


bioRxiv preprint doi: https://doi.org/10.1101/2020.10.27.358077; this version posted July 9, 2021. The copyright holder for this preprint (which was not certified by peer review) is the author/funder, who has granted bioRxiv a license to display the preprint in perpetuity. It is made available under aCC-BY-ND 4.0 International license.

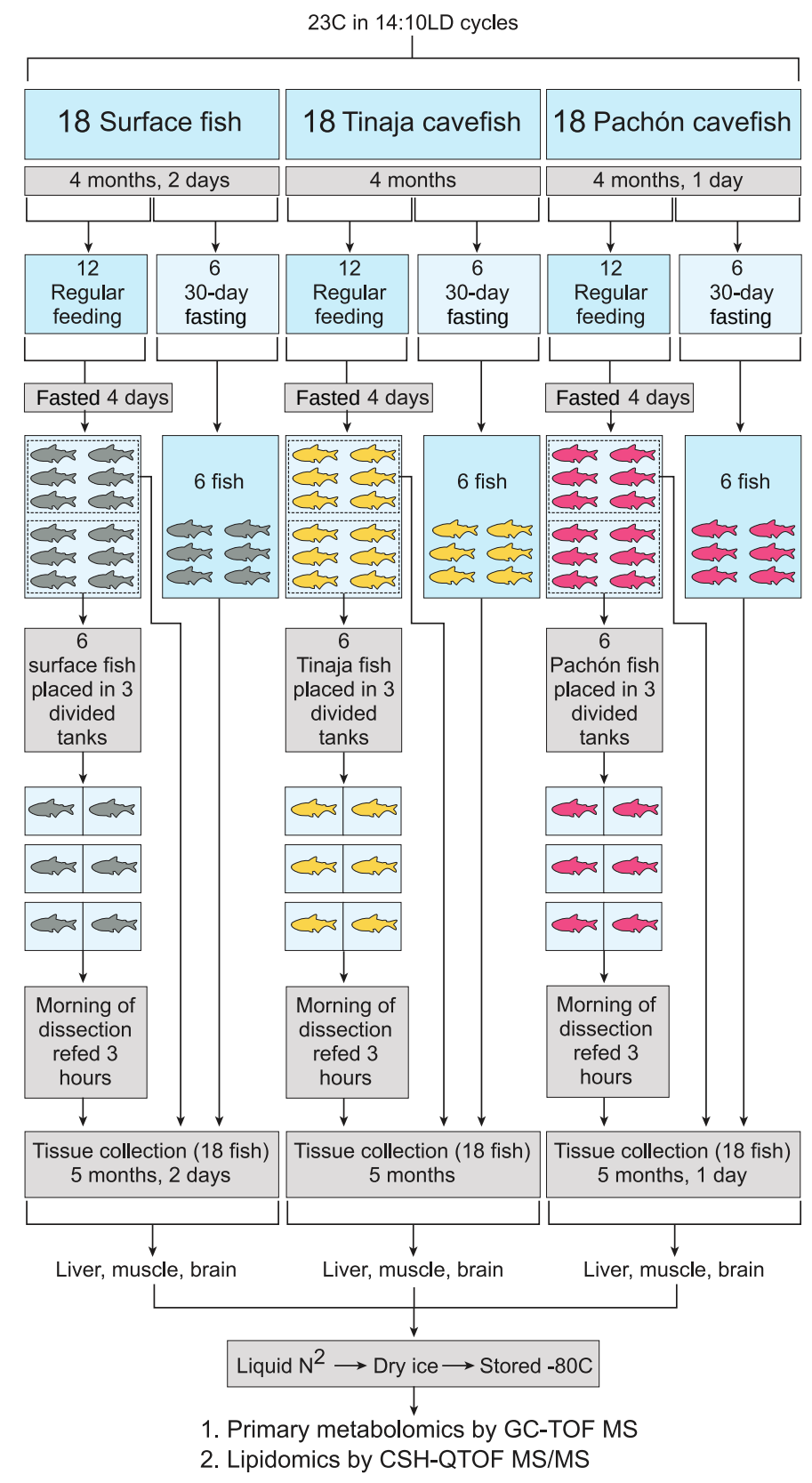

Fig. 2. Experimental setup (A) and PCA for each tissue and metabolite category. Pachón, Tinaja, and surface $A$. mexicanus fry were raised for 4 months and then separated evenly into fasted (30-day) and non-fasted groups. At 4 days prior to collection, non-fasted fish were again divided into two groups (6 fish each) and either fasted for the remaining 4 days (first group) or fasted for 4 days and refed 3 hours prior to collection (second group). Thus, 6 fish were obtained for each of the following conditions: $30-$ day fasting, 4-day fasting, and 4-day fasting followed by re-feeding.

shown that certain health effects, particularly cardiovascular disease, can be predicted from metabolic signatures prior to clinical manifestations. This suggests that metabolic dysregulation has causal influence over the disease state of an organism, and conversely disease may be preventable via metabolic intervention (6).

An evolutionary system with particularly extreme changes in metabolic regulation is the Mexican tetra, Astyanax mexicanus, which has undergone considerable physiological and behavioral changes to colonize a number of subterranean caves in the Sierra de El Abra region of Mexico. Cavefish have evolved a suite of metabolic phenotypes to cope with the cave environment, including lower metabolic rate, increased appetite, fat storage, and starvation resistance $(1,2,7)$. Cavefish are also insulin resistant, hyperglycemic, and exhibit increased caloric intake (3), a feature often associated with decreased longevity. A notable genomic feature in Pachón and Tinaja cavefish is a mutation in the insulin receptor (3) that, in humans, is linked to Rabson-Mendenhall (RM) syndrome, a form of severe insulin resistance that causes many developmental abnormalities and typically progresses to ketoacidosis (8). Nevertheless, cavefish do not appear to suffer any of the adverse effects of RM, lack advanced glycation end products (AGEs) (3) normally associated with hyperglycemia, and live long, healthy lives without ill-effects of metabolic disease (3). A. mexicanus may provide natural solutions to overcome 
A

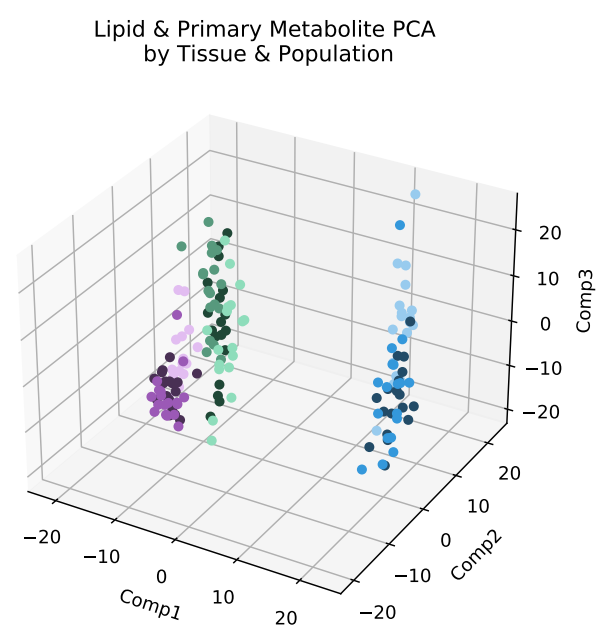

B

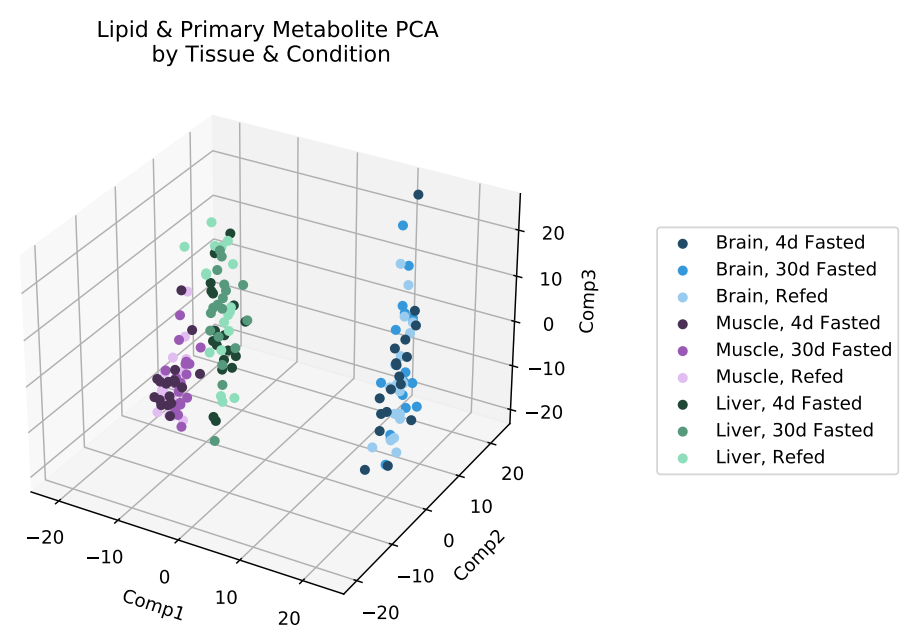

C

- Brain, Pachon

- Brain, Tinaja

- Brain, Surface

- Muscle, Pachon

- Muscle, Tinaja

- Muscle, Surface

- Liver, Pachon

- Liver, Tinaja

- Liver, Surface

D

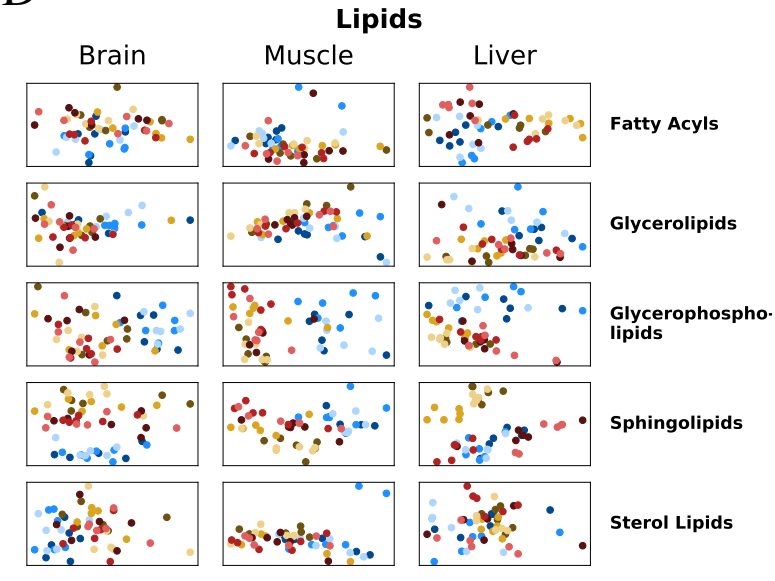

Primary Metabolites

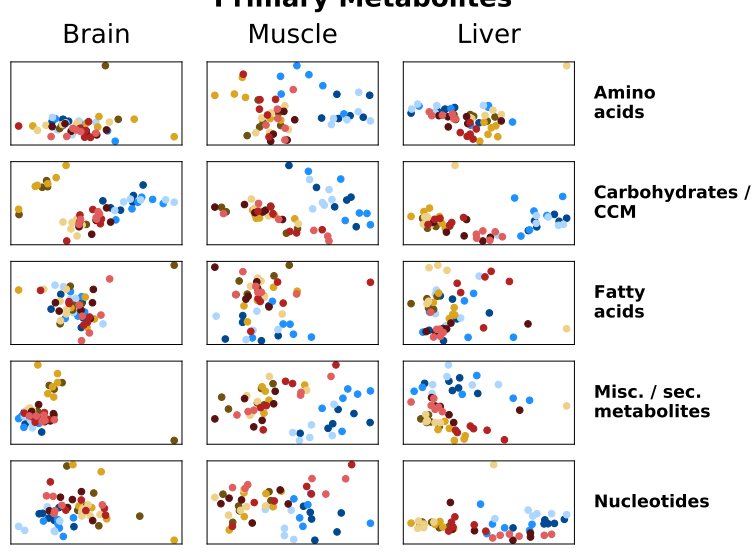

E

- Surface, 4d Fasted
Surface, 30d Fasted
Surface, Refed
Tinaja, 4d Fasted
Tinaja, 30d Fasted
Tinaja, Refed
- Pachon, 4d Fasted
- Pachon, 30d Fasted
Pachon, Refed

Fig. 3. Global trends in lipid and primary metabolite data. To visualize overall patterns in the metabolome of different experimental groups, we performed principal component analysis (PCA) first on all lipids and primary metabolites (A,B), then on individual categories thereof (C,D). Specifically, PCA of mTIC-normalized log-peak intensities of combined lipid and primary metabolite data (filtered by peaks matching either a KEGG compound or a valid LIPIDMAPS id): (A) Samples were colored according to tissue (blue, purple, green) and population (shading of the primary color). (B) Shading by feeding state instead. (C) First (X-axis) and second (Y-axis) PCA components of primary metabolite data subset by five main categories (see Methods) for brain, muscle, and liver of all three populations. (D) PCA of lipid data subset by LIPIDMAPS categorical designation for brain, muscle, and liver of all three populations. (E) Legend for C and D.

the challenges associated with metabolic diseases, like diabetes (9).

From an evolutionary standpoint, survival in the cave environment requires more than starvation resistance, and we use the term metabolic resilience to describe the property of cavefish to withstand a variety of metabolic stresses, including starvation at times and at other times the accumulation of potentially harmful energy metabolites such as triglycerides, glucose, and various derivatives thereof. We hypothesize that survival in the cave environment thus requires multiple, counterbalancing evolutionary changes and that the combined effect of these changes is to make cavefish resilient to a variety of metabolic conditions, of which starvation and triglyceride / sugar accumulation are discrete examples. 

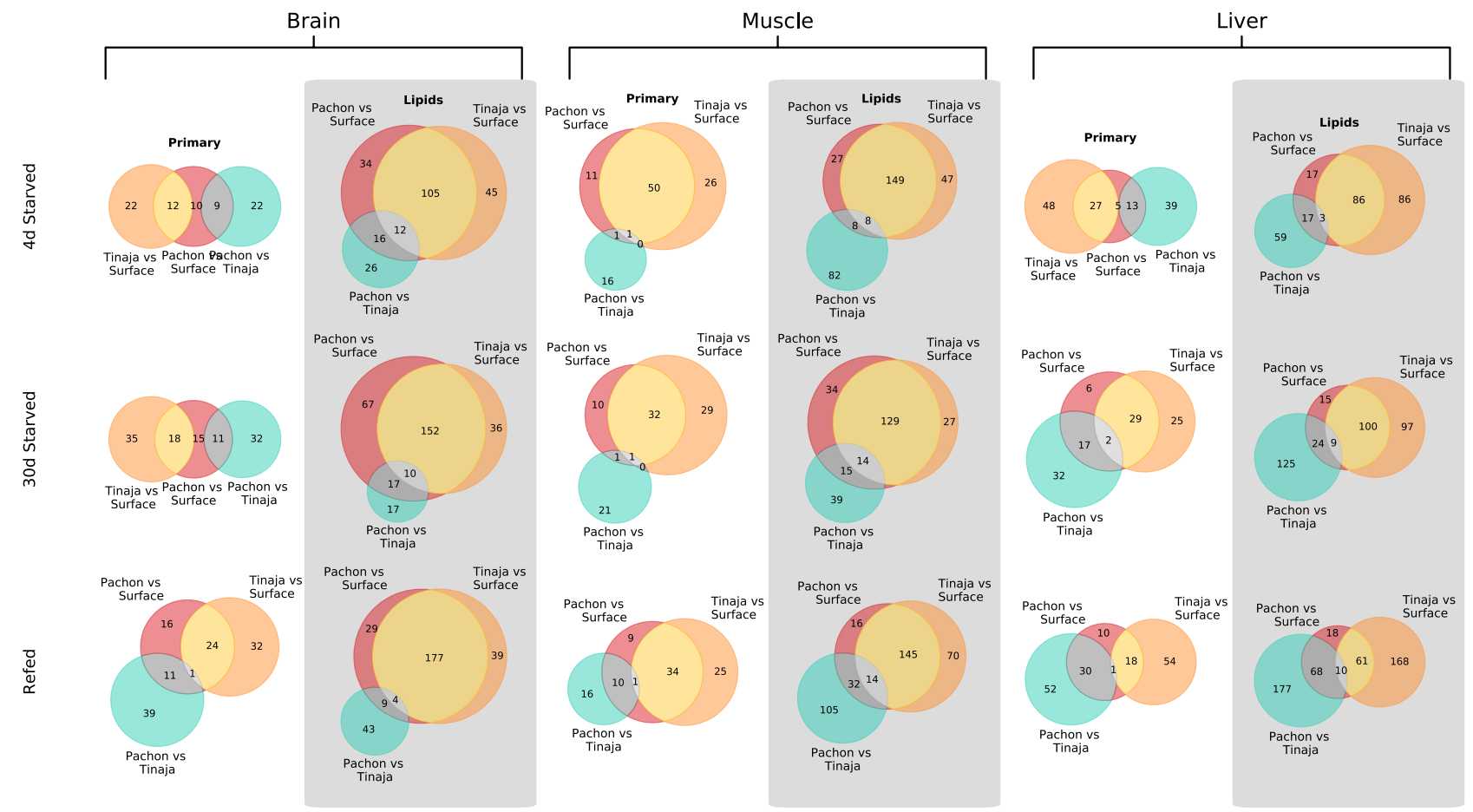

Fig. 4. Common signatures of primary metabolites and lipids in cave populations. In order to determine the degree of agreement between differentially abundant metabolites in Pachón and Tinaja cave populations, we fit an O-PLS / Bayesian GLM statistical model to filtered peak intensity data. The number of metabolites (primary metabolites and lipids respectively) significant at the $p=0.05$ level are shown. Metabolites in the intersection of two or more sets are constrained to agree on a directional basis (i.e. the differential abundance of a given metabolite in both cave populations must be either greater or lesser than surface, not a mixture).

We thus sought to characterize the metabolic signature of resilience by examining the metabolome of Pachón and Tinaja cavefish (two independently-evolved cave populations) compared to surface-dwelling populations using untargeted mass spectrometry (MS) of primary metabolites and lipids. We characterized the response of energetically important tissues (the liver, muscle, and brain) of each population in short/long-term fasted, and fed conditions. We demonstrate that metabolite profiles in Pachón and Tinaja cavefish are more similar to each other than surface fish in each feeding state / tissue combination, highlighting the role of parallel evolution in shaping the metabolome of cavefish. We identify metabolic signatures and metabolites exhibiting the most pronounced regulatory changes within each tissue, population, and feeding state. We constructed inter-population and inter-feeding state comparisons and fit separate statistical models to each case (Fig S1). Cavefish exhibit many similarities with human models of obesity and metabolic syndrome, but also differ from these conditions in terms of antioxidants, metabolites associated with cellular respiration / the electron transport chain, and unexpectedly reduced levels of cholesteryl esters. Moreover, we observe a significant degree of overlap in metabolic strategies, including increased abundance of antioxidants (10) and reliance on sugars as an energy source (11), between cavefish and the long-lived rodent the naked mole-rat (Heterocephalus glaber), suggesting that metabolic resilience in cavefish does indeed include certain features that appear to be consistently associated with robust health and longevity across taxa.

Our results lay the groundwork to explore the mechanistic roles of metabolites and pathways in the adaptation of cave- fish and suggest that natural evolutionary systems may offer insights into metabolic function by showing how disease states can be altered or counterbalanced under a genetic background more suited to a different set of parameters governing states like obesity and starvation resistance.

We also provide a shiny app at https://cavefin. shinyapps.io/shiny to allow others to explore and visualize our dataset.

\section{Results}

Our experimental design aimed to a) characterize the response of the A. mexicanus metabolome to different feeding states in energetically expensive tissues and b) utilize comparisons across populations and feeding states to identify metabolites conserved in cavefish populations. Food scarcity is one of the cave's harshest evolutionary pressures. Cavefish have specialized feeding strategies and fat metabolism that helps them thrive in the cave environment $(1,2,7,12)$. We raised age-matched offspring of Surface (river) fish, and Pachón and Tinaja cavefish morphs originating from two independent cave colonizations. To understand how the cavefish metabolome adapts to ecologically relevant food challenges, we separated Surface, Pachón and Tinaja populations into three different groups at 4-months: 30-day fasted, 4-day fasted, and "Refed" (fed at 3 hours prior to collection after 4days without food) (Fig 2). We chose 4-months so that tissue collection would take place prior to sexual maturity (which occurs at 6-9 months). We made all efforts to equalize the mass and standard length (SL) distributions of fish in each group prior to separation (Fig S2, Table S1, Initial mass and 


\section{Initial SL).}

Our untargeted metabolomics study yielded a total of 174 identified metabolites linked to KEGG (13) / HMDB (14) IDS and 483 identified lipids linked to LIPIDMAPS IDS. We examined the effect of normalizing identified peak values by the total sum of peaks (mTIC) and by sample weight (Fig S3) and found that mTIC is more robust to variations in sample weight. Hence, we employed mTIC-normalized data for the remainder of the analysis.

Figure $3 \mathrm{~A} / \mathrm{B}$ shows that metabolites cluster primarily by tissue, in line with previous studies in mammals (15). Figure $3 \mathrm{C} / \mathrm{D}$ shows how clustering patterns depend strongly on the chemical classification of identified metabolites. Some lipid and primary metabolite categories show a clear separation between different populations (e.g. carbohydrates, Fig 3C, and glycerophospholipids, Fig 3D, across most tissues), whereas other categories have a less pronounced change (amino acids in the brain, Fig 3C, and fatty acyls in most tissues). In order to quantify separation of feeding states as a function of population and metabolite category, we used a supervised machine learning method based on orthogonal projection of latent structures (O-PLS, Fig S4). We then used O-PLS to remove "orthogonal" variation (16) from each metabolite category and fit a Bayesian logistic regression model to the denoised data (Supplemental Methods).

\section{Common Metabolite Signatures and Adaptive Re-} sponse In general, the metabolome of all three populations shows a large degree of similarity within a given tissue (Fig S5), highlighting the influence of genetic ancestry, even in subpopulations that show markedly different phenotypes.

To quantify the degree to which the metabolomic signature of Pachón and Tinaja cave populations is conserved, we computed the directional intersection of metabolites either increased or decreased (but not a mixture of both) in Pachón and Tinaja with respect to surface according to the GLM (Fig 4). In other words, metabolites in the yellow wedge represent the intersection of metabolites that have significant differential abundance in Pachón (red circle) and Tinaja (gold circle) and are increased in both Pachón and Tinaja or decreased in both Pachón and Tinaja.

A large convergent signal was displayed between Pachón and Tinaja cave populations, with the differences between cave populations typically being fewer than the differences between each respective cave population and surface. Muscle displayed the highest degree of common primary metabolites, and both muscle and brain display a large overlap of differentially abundant lipids. Given that Pachón and Tinaja represent independent cave populations, adaptive shaping of the metabolome thus appears to have the strongest effect in muscle, the site of energy expenditure during locomotion, and the brain, where lipids may play an important role in signaling. Thus, adaptation appears to play at least a partial role in shaping the metabolome of cave-dwelling A. mexicanus. Adaptation is particularly highlighted in certain classes of metabolites, which display extreme changes in both cave populations, as described in the following subsections.
Sugar Phosphate Metabolism Given the overall similarity at the tissue level for most classes of metabolites (Fig S5), phenotypic differences are likely to be linked to a relatively small subset of the metabolome. We sought to identify metabolites that could be responsible for the drastic change in phenotype of cave populations. Sugars and sugar phosphates are important energy metabolites and hence candidates for adaptations related to resistance to nutrient deprivation. This class of metabolites displays a dramatic change during shortand long-term fasting, particularly in the liver (Fig 5). Hepatic glucose production is derived from gluconeogenesis and glycogenolysis, the latter relying on stored glycogen, which is quickly exhausted during fasting (17), indicating that hepatic gluconeogenesis likely plays a role in sustaining survival under long-term nutrient deprivation in A. mexicanus. Surprisingly, surface fish also show stable (albeit generally lower) sugar levels in the liver under different feeding states (Fig 5), indicating that sugar production in the liver may be driven by overall demand rather than supply. This may point to a shift from oxidative to sugar-based metabolism as an energy source in energetically expensive tissues. Cavefish possess a larger amount of body fat $(1,2)$, and hence have a larger pool of glycerol to serve as a substrate for gluconeogenesis. We find that both cave populations exhibit decreased levels of glycerol in the 30-day fasted state, particularly in Tinaja (Table S2), indicating increased consumption of this intermediate as a substrate for gluconeogenesis may be the source of increased sugar / sugar phosphate abundance in cave populations.

Regardless of the substrates leading to sugar metabolite accumulation, it is clear that a large difference exists between cave and surface populations within this class of metabolites. However, the specifics of this alteration to sugar metabolism appear to be population-specific, with Tinaja showing a large increase in sugar phosphates and Pachón showing an increase in unphosphorylated sugars respectively in short/long-term fasted states in comparison to surface (Fig 5).

Other tissues show a mixed response, with muscle displaying increased levels of most sugar phosphates in Pachón but decreased levels of fructose-1-phosphate in both cave populations with respect to surface. The brain displays low levels of sugar / sugar phosphate metabolites overall but possesses increased sugar metabolite abundance in cave populations for certain metabolites and feeding states (Table S2). In particular, fructose and fructose phosphates tend to be upregulated in the brain, suggesting that reliance on glycolytic metabolites may be a strategy used by cavefish to survive in low nutrient or hypoxic environments, an adaptation that is also present in naked mole-rats (11).

While the levels of most simple sugars and sugar phosphates are increased in cavefish with respect to surface, the levels of gluconic acid and glucoronic acid show the opposite pattern (Fig 5). Gluconic acid and glucoronic acid belong to uronic acids, a class of sugar acids that are major building blocks of proteoglycans. It has previously been observed that A. mexicanus cave morphs lack advanced glycation end products (3), which are a defining feature of diabetes and are nor- 

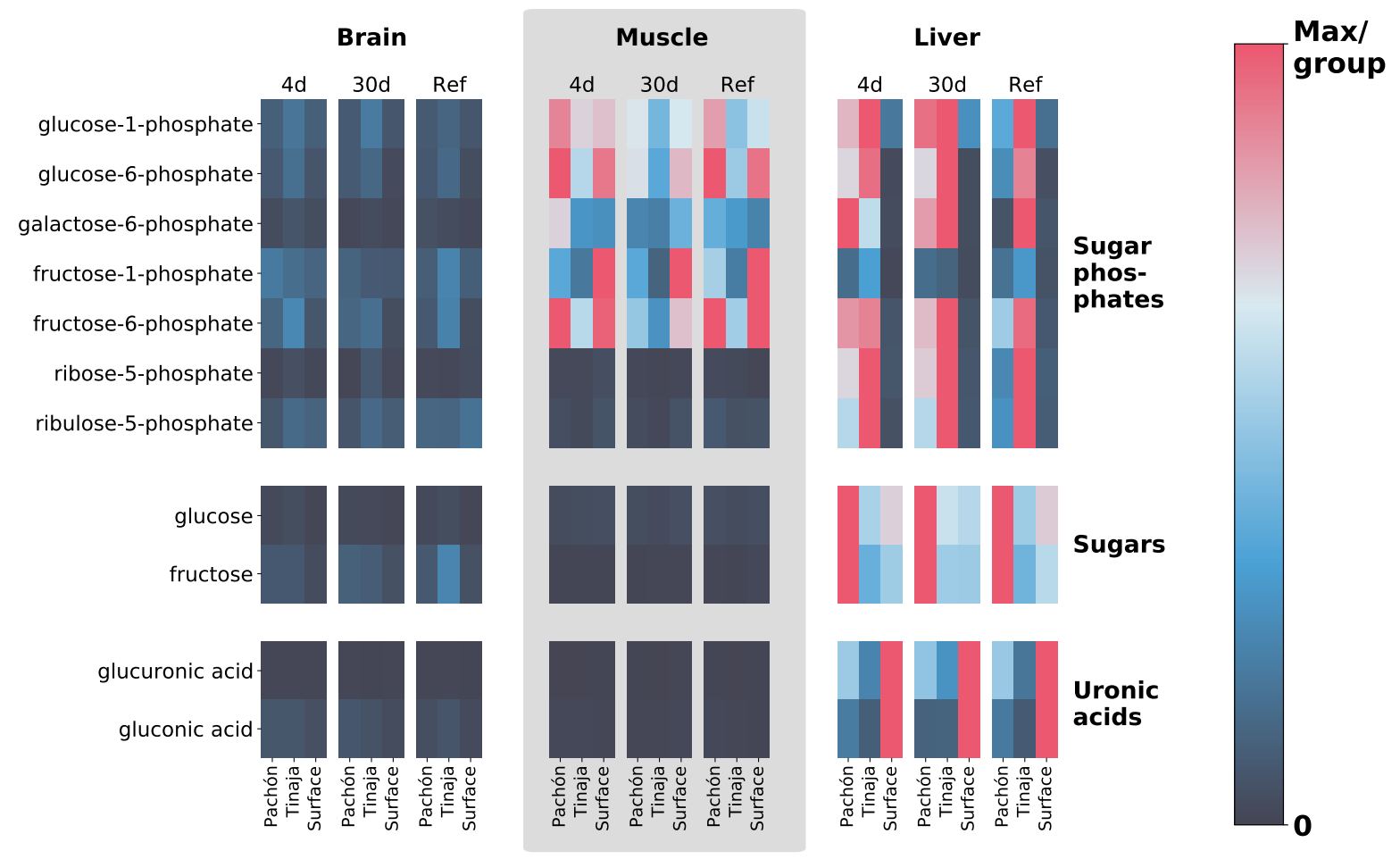

Fig. 5. Extreme alterations to sugar metabolites in cave vs. surface populations. To show differences in cavefish sugar metabolism, we selected 5- and 6-carbon sugars, sugar phosphates, and uronic acids (oxidized forms of sugars that form building blocks of proteoglycans). The row axis shows metabolites within each of these classes, and the column axis shows different populations (short range, labels at bottom), feeding states (medium-range, labels at top) and tissues (long-range, labels at topmost point). Color indicates the mTIC-normalized peak intensity for the average of 6 biological replicates. Red indicates the maximum value for a given row (i.e. across all populations, tissues, and feeding states), whereas navy blue (the bottom of the color bar) corresponds to a peak intensity of zero, not to the minimum value. Thus, dark cells correspond to very little / zero intensity, as opposed to simply corresponding to the minimum intensity within the row. Given that mTIC intensities are "semi-quantitative", red values thus correspond to the most abundant group for a given metabolite, and navy blue corresponds to lack of abundance.

mally associated with chronic hyperglycemia in humans. Altered metabolism of sugar acids in cavefish may play a role in inhibiting excessive protein glycation and the adverse health effects thereof.

Ascorbate A highly unexpected and unexplained feature of our analysis is the abundance of vitamin C, particularly in its oxidized form dehydroascorbic acid (DHAA), across all tissues in cave populations (Fig 6). Ascorbic acid (AA), the reduced, active form, is also more prevalent in muscle tissue. DHAA can be recycled back to AA using reducing cofactors such as NADH and NADPH, which can in turn be regenerated from the pentose phosphate pathway and TCA cycle using simple sugars (which cavefish possess in great abundance). For this reason, vitamin $\mathrm{C}$ content in food labeling is usually reported as the sum of AA and DHAA (20). Thus, cavefish possess a larger total 'pool' of vitamin C (including interconvertible oxidized and reduced forms, Fig 6).

Many cavefish populations exhibit increased appetite and carry an allele of the melanocortin 4 receptor that predisposes them to hyperphagia (1). The increased appetite could cause cavefish to consume more overall food, which could be responsible for the AA/DHAA increase in the refed state. However, this does not pertain to the 30-day fasted state, where AA/DHAA levels are also elevated across all tissues. There is widespread consensus that teleosts, like humans, lack the ability to produce AA endogenously due to the absence of gulonolactone oxidase, which catalyzes the final step in AA biosynthesis (21). In humans, this enzyme is a pseudogene, whereas in teleosts the gene is absent entirely, thought to be lost in the distant evolutionary past. Thus, the additional AA/DHAA supply likely comes from selective reuptake in the kidney, a process that also occurs in humans to conserve AA/DHAA, or it may be produced by commensal microbiota in cavefish. Trace amounts of AA/DHAA in the feed used in the aquatics facility used to house the fish in this experiment may recirculate throughout the water filtration system and be redistributed to all tanks, including those housing fish in the fasted groups. Nevertheless, it remains that even in the case of circulating trace amounts of AA/DHAA, cavefish appear to exhibit selective retainment of AA/DHAA in larger quantities.

The advantages of AA conservation in adaptation to an environment where prolonged starvation is common are selfevident. AA is involved in collagen formation, and its deficiency leads major loss of integrity of connective tissue. Thus, the ability to retain what little ascorbate is present in underground cave environments would confer an enormous survival advantage to fish.

Another factor that could influence the AA/DHAA ratio is the effect of insulin resistance and hyperglycemia on the GLUT family of transporters, particularly GLUT4 in adipose / muscle tissue $(20,22)$. DHAA competes with glucose for transport across the membrane by GLUT4, whereas AA is taken up by $\mathrm{Na}^{+}$transporters. GLUT4 activity is dependent on membrane translocation and this process is dysregulated in 
A
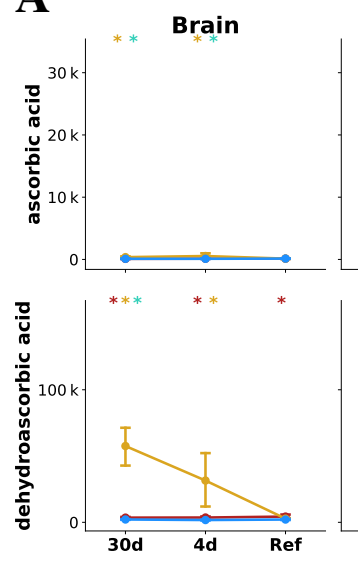

Liver

C
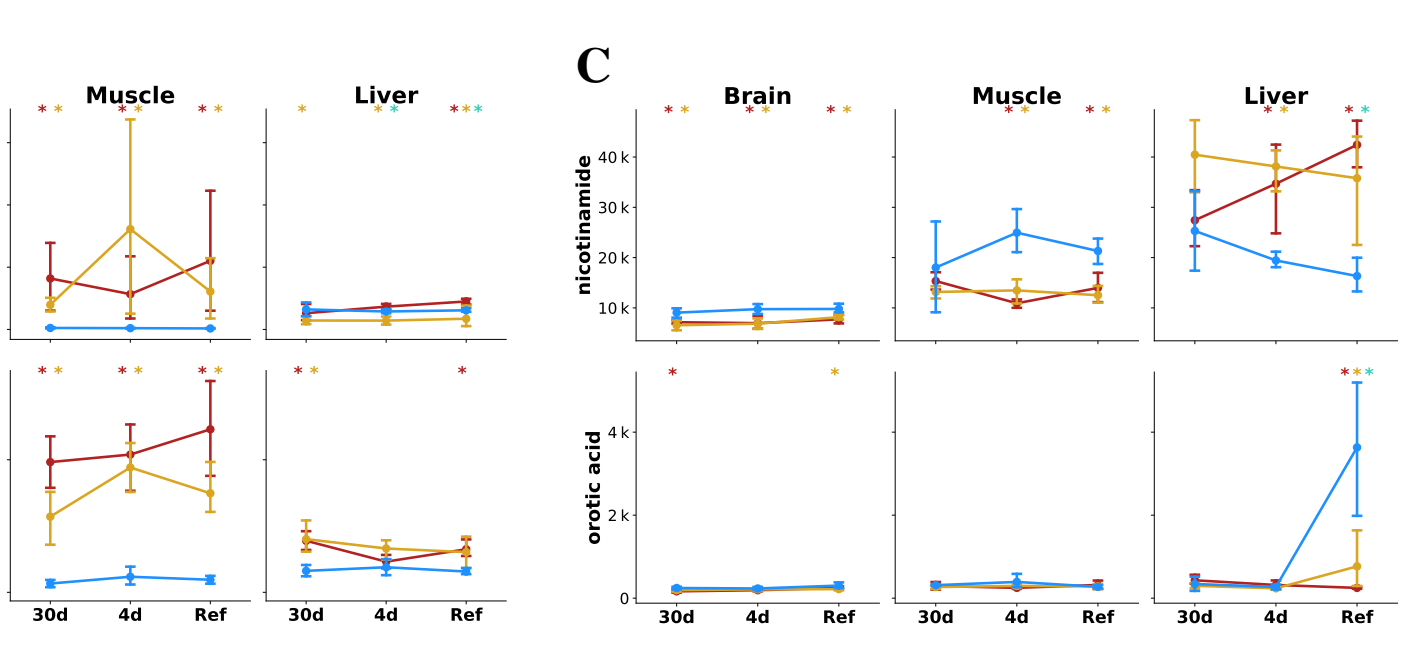

B

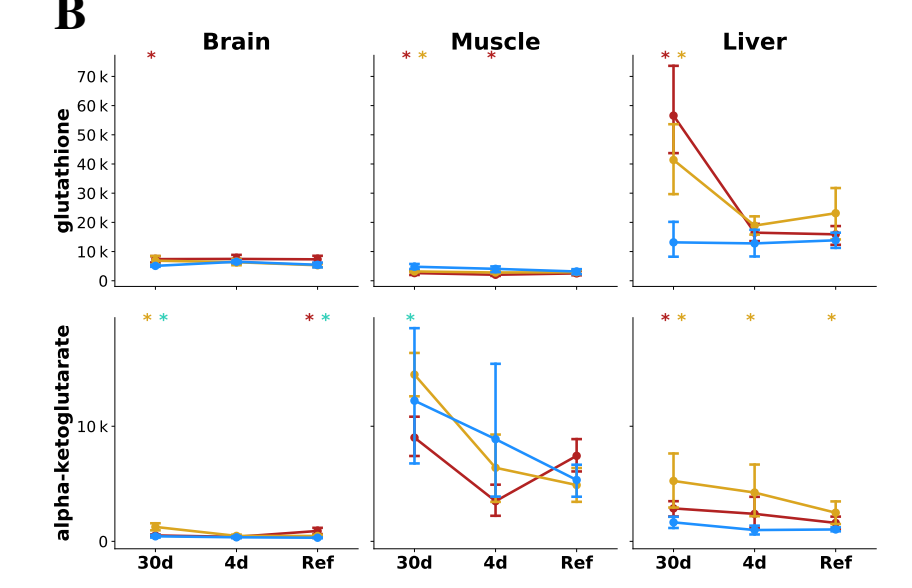

bioRxiv preprint doi: https://doi.org/10.1101/2020.10.27.358077; this version posted July $9,2021 . T$
was not certified by peer review) is the author/funder, who has granted bioRxiv a license to disp
available under aCC-BY-ND 4.0 International license.

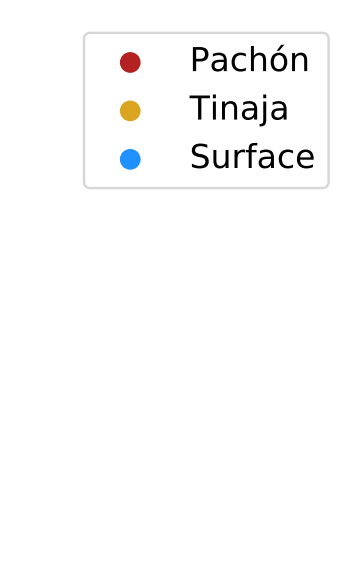

Fig. 6. The cave and surface fish metabolome highlights differences in antioxidant availability and oxidative metabolism. To analyze changes in redox metabolism in cave populations, we compared the abundance of potent antioxidants and redox-linked metabolites. Metabolites are plotted as mTIC-normalized peak intensities (A,B,C, error bars indicate 2.5 / 97.5 percentiles). Asterisks indicate significance at the 0.05 level according to an O-PLS / Bayesian logistic regression (Methods) for Pachón vs surface (*), Tinaja vs surface (*), and Pachón vs Tinaja (*). (A) Ascorbate (vitamin C) is a potent antioxidant and essential nutrient. Vitamin C exists as the reduced form ascorbic acid (AA) and oxidized form dehydroascorbic acid (DHAA), which can interconverted by cellular processes. (B) Glutathione, another antioxidant, is significantly increased in the liver and brain under 30-day fasting. Alpha-ketoglutarate is a tricarboxylic acid cycle (TCA) intermediate that has been linked to longevity in nematodes and mice (18, 19). (C) Nicotinamide is a precursor to $\mathrm{NAD}^{+}$synthesis via a salvage pathway, and increased in the liver but decreased in other tissues in cavefish. Orotic acid is a metabolite that causes fatty liver disease in rats when added to a chow diet.

diabetes (23). This combination of elevated blood sugar and insulin resistance suggests that GLUT4 could be less active in cavefish and cause DHAA to accumulate in the extracellular space. AA/DHAA have also been reported to influence C-reactive protein levels (24) and protein glycation (25), prompting further investigation into A. mexicanus as a model of diabetes-related adaptations. Finally, Pachón cavefish possess a reduction in neutrophils, one cell type which are normally involved in the uptake of AA and reduction to DHAA, compared to surface (26).

Adaptation to Hypoxic Conditions Energy metabolism in most organisms can be viewed as a balance between oxidative processes (cellular respiration via oxidative phosphorylation and the electron transport chain) and sugar metabolism, and the relative contributions of these two processes can have important physiological consequences, as in the wellknown Warburg effect in cancer. A. mexicanus cave morphs have considerably upregulated sugar metabolism (Fig 5), and also display decreased levels of several products of oxidative metabolism. One important metabolite in this category that displays differences in cave populations is $\alpha$-ketoglutarate $(\alpha-\mathrm{KG})$, which has increased abundance in the liver in all feeding states and in the brain in certain feeding states in both cave populations. $\alpha$-KG supplementation has been linked to lifespan extension in C. elegans (18) and mice (19). Furthermore, uronic acids, the oxidative products of simple sugars, are significantly reduced in the liver of both cave populations (Fig 5, Table S2), suggesting that troglomorphs are characterized by decreased reliance on oxidative metabolism and increased reliance on sugar metabolism. This would enable cavefish to survive in a hypoxic subterranean environment, similar to naked mole-rats (11).

Obesity and Inflammation-Related Metabolites In humans, chronic inflammation of adipose tissue is a common feature of obesity and can often lead to insulin resistance and eventually type 2 diabetes (27). Cave populations of A. mexicanus have been previously reported to exhibit pronounced insulin resistance (3), but do not accumulate advanced glycation end products and do not appear to have diminished longevity. 

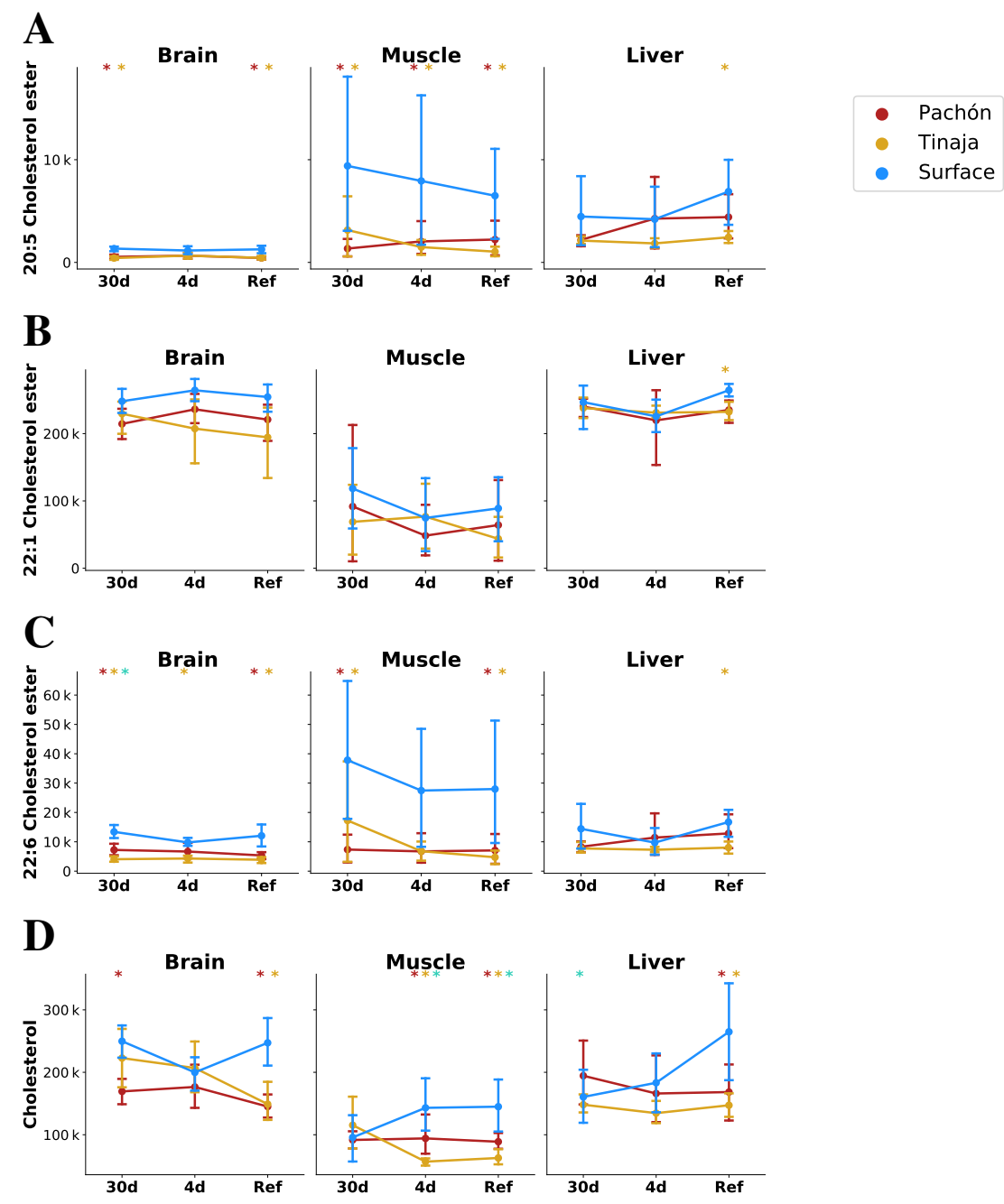

Fig. 7. Parallel evolution in cavefish exhibits profound alteration of cholesterol / cholesteryl ester metabolism. Cavefish possess a significant reduction in certain long-chain fatty acid cholesteryl esters (A-C) and cholesterol itself (D), particular in peripheral tissues (muscle). Values on the y-axis are mTIC-normalized peak intensities for each lipid species. Asterisks indicate significance at the 0.05 level according to an O-PLS / Bayesian logistic regression (Methods) for Pachón vs surface (*), Tinaja vs surface $(*)$, and Pachón vs Tinaja (*).

In order to compare the metabolome of A. mexicanus cave populations to the known metabolic signatures of obesity (5), we calculated changes in lipid categories (the coarsest abstraction used in LipidMaps; Table S3), classes (a more detailed partitioning scheme used in LipidMaps; Table S4), and, within free fatty acids specifically, the degree of saturation (Table S5). Additionally, we also examined a subset of the lipid data corresponding to individual lipid species with common names (Table S6). The metabolome displays a remarkable overlap with the proinflammatory signature associated with obesity that, in humans, leads to insulin resistance. This signature consists of (1) the elevation of saturated fatty acids (SFAs) in muscle in most feeding states (Table S5), which have a direct and pronounced proinflammatory effect in mammals through the recruitment of macrophages (28) (although the importance of fatty acid release in insulin resistance is disputed $(29,30)$ ), (2) abundance of ceramides in muscle in all feeding states (Table S4), which are known direct mediators of insulin signaling (28). Indeed, the only feeding state for which skeletal muscle did not display increased SFA abundance was 30-day fasting, which could sim- ply indicate the exhaustion of free SFA pools. Additionally, palmitate, a precursor of ceramide biosynthesis (28), is elevated in muscle in all feeding states (Table S6). Sphingoid bases are significantly more abundant in muscle in all feeding states (Table S3), suggesting generally upregulated sphingolipid biosynthesis in cave populations.

In contrast to proinflammatory metabolites, omega-3 fatty acids ( $\omega-3$ FAs) such as DHA and EPA have protective effects against inflammation $(27,28,31)$. These molecules bind to the GRP120 receptor on macrophages and adipocytes, and the activated receptor then modulates the activity of PPAR $\gamma$ and ERK $(27,31) . \omega-3$ FAs are less abundant in the liver under 4-day fasting (Table S6) and are generally not upregulated in most feeding states and tissues (Table S6). Thus, $\omega-3$ FAs do not appear to offset for the proinflammatory signature of cavefish SFA and ceramide signatures, suggesting that cavefish possess an alternate compensatory mechanism to prevent chronic tissue inflammation. Overall, cavefish appear to exhibit many metabolic similarities with obesity and health conditions associated with it.

However, this is not a universal trend. Cirulli et al. report a 
A
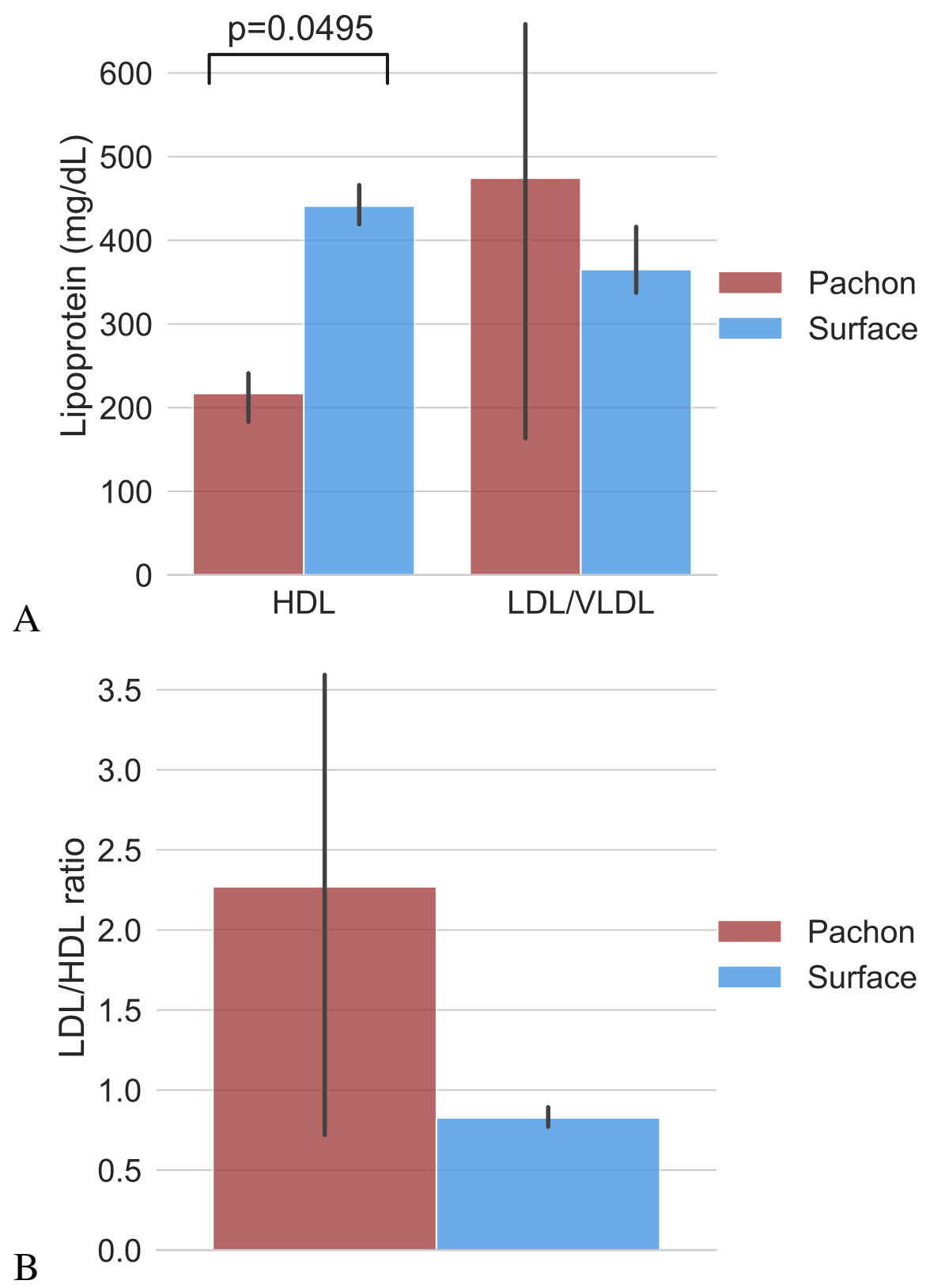

Fig. 8. Adult Pachón cavefish possess lower HDL than surface fish. (A) A colorimetric assay (see Methods) was used to measure lipoprotein levels in Pachón and surface populations. Absolute levels of both HDL and LDL/VLDL appeared to exhibit differences between populations, but only HDL was found to be significant via a Kruskal-Wallis test. (B) The LDL/HDL ratio was computed by dividing LDL/VLDL brightness measurements by HDL brightness measurements and showed a large degree of variability in Pachón LDL/VLDL fraction and hence was not statistically significant across populations, although a large relative difference in means exists. Raw data are given in Table S12.

strong association between urate levels and BMI, likely due to insulin resistance interfering with uric acid secretion in the kidney (5). In contrast, cavefish appear to have significantly reduced levels of uric acid in muscle, and in other tissues levels are comparable with surface (except for a small but significant increase in Pachón liver during fasted states, Table S2). Uric acid is increased in humans during prolonged fasting (32), but this trend again does not hold for cavefish, particularly Tinaja. Mannose, which is associated with obesity and insulin resistance in humans (5), was abundant in the Pachón liver in all feeding states, but was reduced in Tinaja compared to surface fish.
Finally, cholesteryl esters (Table S6), and cholesterol in some feeding states (Table S2), were less abundant in cave populations. Using a previously published gene expression dataset (33), we investigated factors that might influence levels of cholesteryl esters. Cholesteryl ester transfer protein (CETP), which transports cholesterol in / out of lipoproteins, is downregulated in both Pachón and Tinaja compared to surface fish (Fig S6), suggesting a potential causal relationship between lower cholesteryl ester levels in cave populations and this important carrier protein. A human variant of CETP associated with decreased serum levels of the protein and larger low-density lipoprotein (LDL) and high-density lipoprotein 
(HDL) particle sizes has been linked to exceptional longevity (34). The LDL / HDL cholesterol ratio, mediated in part by CETP (35), is a major contributor to risk of atherosclerosis and coronary heart disease (36). The reliance on triglycerides as an energy source in cavefish may increase the risk of arterial disease by providing an abundance of free fatty acids and other lipids. Cholesteryl esters, in particular, are formed from esterification of a fatty acid and cholesterol, are a major constituent of foam cells in atherosclerotic lesions $(37,38)$, and, for certain lipid species, show a large difference in abundance between cavefish and surface (Fig 7). Inhibition of CETP has been shown to reduce cardiovascular risk, ostensibly by altering the partitioning of cholesteryl esters between LDL and HDL (39). Indeed, lipid homeostasis in zebrafish exhibits strong similarities with human (40), and zebrafish express CETP whereas other model organisms such as mice do not. This raises the question of whether CETP and its cholesteryl ester substrates may be a locus of positive selection in cavefish to offset the risks associated with increased visceral fat.

Large differences in CETP expression suggest that lipid homeostasis may be regulated differently between cave and surface populations. Lipoproteins, in the form of HDL, IDL, LDL, VLDL and chylomicrons contribute to and regulate lipid homeostasis. In order to investigate whether lipoproteins display any significant differences between cave and surface populations, we measured HDL and LDL/VLDL levels between aged Pachón and surface individuals using a colorimetric assay (Fig 8A). LDL/VLDL levels were highly variable in Pachón, but HDL levels showed a consistent difference (Fig 8A). Surprisingly, Pachón cavefish possess an LDL/HDL ratio similar to human (although slightly lower), whereas surface possess higher HDL and lower LDL/VLDL.

In humans, HDL is widely believed to play a protective role in atherosclerosis. However, antioxidants may also influence the risk of atherosclerosis by inhibiting the production of oxidized LDL $(41,42)$. Our discovery of elevated antioxidant levels in cavefish may offset the decreased HDL abundance in this population, and may allow cavefish to tolerate lower plasma HDL levels.

Given alterations in lipid metabolism, we asked whether either cave or surface populations also exhibit signs of increased arterial disease risk as indicated by the presence of arterial lesions. In a separate experiment, we imaged caudal vein sections using Van Gieson staining (c.f. (43)) in order to investigate whether cavefish show signs of arterial lesions at an early life stage. We did not observe arterial lesions in any of our cave or surface samples (Table S10), in stark contrast to zebrafish fed a high cholesterol diet, in which fatty streaks can be readily observed (43).

In summary, lipid metabolism in A. mexicanus represents a hub of evolutionary activity which clearly separates surface and cave populations. Cavefish must balance increased demands on energy storage with counter-adaptations to protect against pro-inflammatory and atherogenic metabolites. We observe elevated levels of most energy metabolites, with the notable exception of cholesterol and cholesteryl esters, and cavefish have a larger (V)LDL/HDL ratio as compared to surface fish. LDL and VLDL have higher triglyceride content as compared to HDL (44) and VLDL is a substrate for lipoprotein lipase (45), which liberates free fatty acids from triglycerides. LDL/VLDL may thus be important for energy metabolism in cavefish, and hence selective pressure may contribute to the higher $(\mathrm{V}) \mathrm{LDL} / \mathrm{HDL}$ ratio in this population.

Resistance to Nutrient Deprivation In order to determine the basis of cavefish adaptation to low-nutrient environments, we sought a statistical test that would be sensitive to metabolites that change significantly between refed and short/longterm fasted states and insensitive to metabolites that remain relatively stable across feeding states. We further hypothesized that certain metabolites may have an important role in cave adaptation. Pachón and Tinaja represent independently evolved populations, and we reasoned that a test for parallel adaptation should be selective for metabolites that show the same differential feeding state response pattern across cave populations (e.g. differentially increased in both Pachón and Tinaja fasted states relative to surface). In order to construct this test, we fitted a Bayesian GLM (46) to a linear combination $(P+T) / 2-S$ of O-PLS-filtered z-score values (see Methods), where $P$ stands for Pachón, $T$ stands for Tinaja, and $S$ stands for surface. We used this test to identify metabolites that might have a role in the fasting response of cavefish, i.e. metabolites that are differentially abundant in cave populations in the fasted versus refed state (Pachón and Tinaja are assigned equal weight), and generally show the opposite pattern in surface. Figure 9 shows the results of this test for 30-day fasting vs. refeeding (A, which corresponds to the most extreme experimental groups), and the two other possible comparisons between feeding states $(\mathrm{B} / \mathrm{C})$.

Using this adaptive criterion, we examined which energy metabolites seem to be specifically correlated with different fasting states (Tables S7, S8, and S9 show the results of different fasting state comparisons). Sugar metabolites do not appear to exhibit a strong differential feeding state response. However, long-chain fatty acids such as palmitate and stearate (Fig 9C) do show differential abundance between long- and short-term fasting, suggesting that cavefish may rely on increased usage of fat stores in long-term fasting. Furthermore, analysis of the 30-day fasting response in cavefish liver highlights orotic acid (OA, Fig 9A), an intermediate in pyrimidine synthesis that has been implicated in fatty liver condition (47). OA is suppressed in all feeding states in cave populations, but exhibits a sharp spike in refed surface fish (Fig 6).

Steatosis, or non-alcoholic fatty liver condition, can be caused by reduced FA oxidation / secretion, or increased FA update or lipogenesis (48), with the majority of fat accumulation in the liver coming from uptake of non-esterified fatty acids in humans (49). Lipidomics data indicate that free fatty acid content in the 30-day fasted state is lower for both cave populations with respect to surface (Table S3). The higher body fat of cave populations may be due to increased fatty acid synthesis in the liver under conditions of nutrient avail- 
A

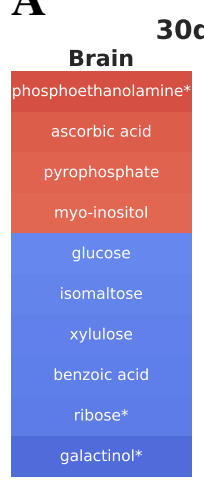

30d Fasted vs Refed

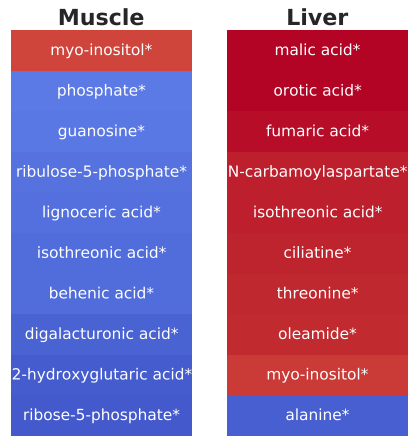

C

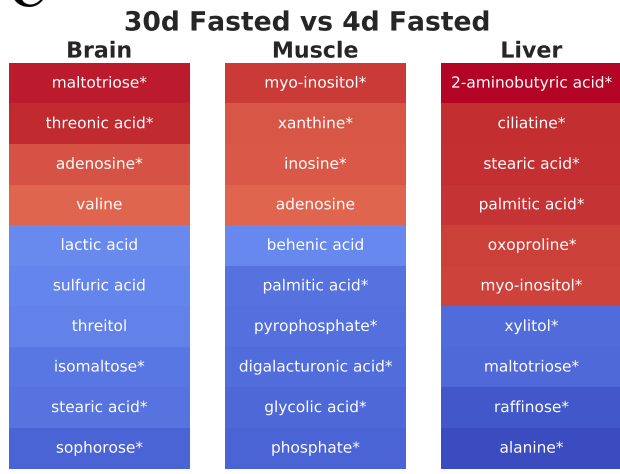

D

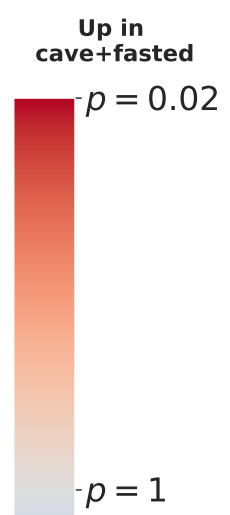

B

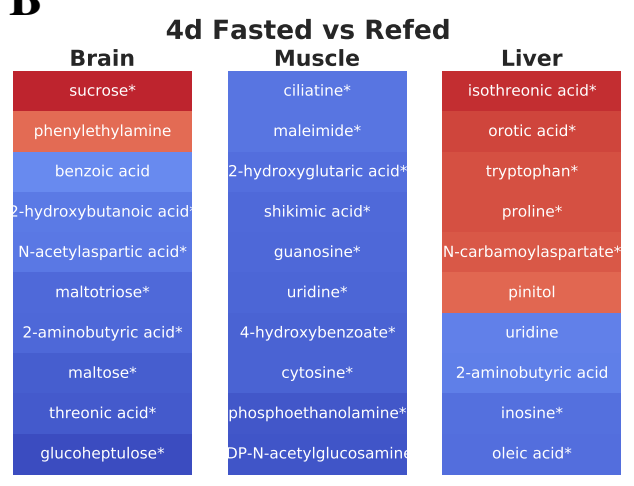

4d Fasted vs Refed

Fig. 9. Parallel adaptive metabolic signature in response to food deprivation in cave populations. To identify metabolites linked to adaptations promoting survival in a nutrient-limited environment shared between cave populations, we fit an O-PLS / GLM statistical model to the response to fasting, i.e. the difference between 30-day fasting and refeeding for Pachón, Tinaja, and surface populations. Specifically, we fitted a Bayesian GLM (see Methods) to the linear combination $(P+T) / 2-S$, with $P, T$, and $S$ referring to the normalized z-scores for each Pachón, Tinaja, and surface sample respectively. Coloring in the figure indicates metabolites increased (red) or decreased (blue) in both cave populations in 30-day fasting (i.e. "up" refers to metabolites that are increased in Pachón and Tinaja in the 30-day fasted state with respect to surface), and color intensity corresponds to $\log _{10}$ p-value, with lighter colors indicating less significant $p$-values and darker colors indicating more significance. The most significant 20 differentially abundant metabolites (regardless of direction) in each tissue for both cave populations with respect to surface are displayed. An asterisk ( ${ }^{\star}$ ) indicates significance at the 0.05 level.

ability. Increased lipid accumulation from this process could render cave populations vulnerable to steatosis / fatty liver condition. Given the role of OA in fatty liver condition, OA suppression may therefore have a potential role in preventing excessive lipid accumulation in the liver of cave populations.

Starvation has detrimental effects on an organism in many ways. One detrimental effect is the depletion of antioxidant substances and the resulting oxidative stress through increasing levels of reactive oxygen species (ROS) (50). Studies that focus on the impact of food deprivation on oxidative stress in fish show that prolonged starvation decreases the capacity of fish to ameliorate oxidative stress (50). Glutathione is a major antioxidant that detoxifies ROS and thereby prevents cellular damage from oxidative stress (51). Cavefish face prolonged periods of nutrient deprivation in their natural environment (1). Adaptation to the cave environment may have led to changes in glutathione metabolism in cavefish to protect against oxidative stress under prolonged fasting. Indeed, in an earlier study we were able to demonstrate that cavefish show an increased expression of genes that are involved in the metabolism of glutathione, which is indicative of an increased stress level compared to surface fish in their natural habitat (4).Here, we can confirm that these trends in gene ex- pression are accompanied by elevation of reduced glutathione in the liver and brain (Fig 6, Table S2). We did not observe a significant increase of glutathione in the surface fish in the fasted states (Fig 6, Table S2).

Guided by these observations, we further attempted to characterize the role of altered antioxidant and cholesterol metabolism in cavefish. In particular, we hypothesized that cavefish resilience to widely varying nutrient levels is driven by a robust antioxidant system that prevents the accumulation of ROS under conditions of stress (e.g. induced by fasting).

To test this hypothesis, we examined ROS state in the liver under a subset of our original fasting experiment using similarly aged fish. ROS accumulation has been shown to cause lethal levels of cell damage in flies, but such damage can be prevented via oral administration of antioxidants or overexpression of antioxidant-producing enzymes (52). This caused us to ask whether naturally elevated antioxidant levels in cavefish might protect against starvation-induced ROS accumulation. We reasoned that the most extreme changes are observed between 30-fasted and 4-day fasted + refed groups, and that given the similarity in Pachón and Tinaja antioxidant profiles, one cave population would be sufficient for validation. We thus repeated the fasting experiment using only $30-$ 
day fasted and 4-day fasted + refed groups, and using only Pachón and surface populations.

Using dihydroethidium (DHE) staining, we examined different populations and feeding states for ROS level. While liver sections of fasted / refed fish did not show differences in cytoplasmic / nuclear localization of DHE (Fig S7B-E), a quantitative analysis showed that, under 30-day fasting, juvenile Pachón cavefish exhibit lower levels of ROS in cytoplasm as compared to surface under the same conditions (Fig S7A), supporting the hypothesis that elevated antioxidants in cavefish may have a protective effect by neutralizing active ROS and may have arisen as an adaptation allowing cavefish to tolerate ROS produced by long periods of nutrient deprivation. We did not observe significant differences in DHE nuclear intensity for either the cytoplasm or the nucleus, suggesting that 30 days of fasting may not be sufficient to produce salient differences in the nucleus for juvenile fish. The known resilience properties of antioxidants may also help explain the metabolic resilience of cavefish by providing a buffer against different forms of stress.

Cavefish thus exhibit decreased ROS abundance in the liver in 30-day fasted conditions, which correlates with the trend we observed in glutathione. Antioxidant levels in cavefish may have evolved as a strategy to allow cavefish to tolerate variation in nutrient availability, as a means of controlling inflammation caused by deleterious energy metabolites, as a way of inhibiting atherogenesis, or several of these effects.

\section{Discussion}

A. mexicanus has been advanced as a model of resilience under ostensibly pathological conditions including hyperglycemia, diabetes (9), and insulin resistance (3). Here, we have provided a large, untargeted study of the metabolome of A. mexicanus surface fish and two cave populations in order to investigate the molecular underpinnings of these adaptations.

We were particularly interested in the role of metabolism in cave adaptation of the two A. mexicanus cavefish populations in this study: Pachón and Tinaja. We found evidence of cavemediated positive selection in the form of overlap of changes in metabolite levels (particularly lipids) in certain tissues under all feeding conditions (Fig 4). This suggests that parallel adaptation to cave environments requires satisfying certain common metabolic needs that are an inherent part of the niche. The obvious candidate for this evolutionary conflux is adaptation to a low-nutrient environment. However, metabolic strategies for survival in such environments are not currently well-understood. We found that drastic alterations in energy metabolism, together with shifts in mediators of redox metabolism and ascorbate, an essential vitamin which is lacking in the cave environment, constitute a major feature of cave adaptation in these populations.

Cavefish appear to have substantially altered sugar metabolism, and exhibit higher levels of sugars and sugar phosphates. However, the opposite trend occurs for uronic acids, which are the oxidized forms of simple reducing sugars and can be formed enzymatically or non-enzymatically.
This incongruency can be resolved by noting the overall trend to decreased reliance on oxidative metabolism (and enzymes that catalyze oxidative processes) and increased reliance on sugar metabolism. This trend stems from sugars and sugar phosphates, antioxidants such as ascorbate and glutathione, and $\alpha-\mathrm{KG}$ (which has been shown to inhibit the electron transport chain in C. elegans). Due to drastic fluctuation in oxygen level in the subterranean niche, cavefish may rely on a shift from oxidative to predominantly sugar-derived energy metabolism, as compared to their surface-dwelling cousins. Reduction in uronic acids, which are derived from sugars using oxidative processes, can thus be seen as part of this trend. However, the specific reduction of uronic acids in particular may have an additional survival benefit for cavefish by inhibiting protein glycation and thus preventing accumulation of advanced glycation end products. Further investigation is required to fully understand the evolutionary and physiological implications of these metabolic changes.

The altered sugar metabolism of cavefish may be indicative of a shift from oxidative to sugar-based energy metabolism, similar to certain metabolic adaptations in naked mole-rats (11). Further work is required to establish the extent of hypoxic conditions in A. mexicanus evolution. However, we also find that certain redox-related metabolites, including $\alpha$ $\mathrm{KG}$, glutathione, and ascorbate, all exhibit distinctive abundance patterns in cavefish. These patterns may be in response to hypoxia, poor nutrient conditions, differences in metabolic rate, or some other aspect of the cave niche.

Our data indicate that upregulation of glucose and longchain fatty acid production is a common feature shared by Pachón and Tinaja cave populations, suggesting that certain cave habitats do require considerable changes in energy metabolism. Pachón and Tinaja likely have a greater reliance on fat stores for locomotion, as evidenced by increased SFA content in muscle in fasting and refeeding. The decrease of $\omega-3$ FAs during fasting (Table S6) coupled with the increase of palmitate (Table S9) in long- vs short-term fasting suggests that cavefish metabolism may be preferentially biased towards storing caloric intake as energy-rich saturated fatty acids. Whether cavefish possess adaptations to counteract the deleterious effects of high body fat, such as suppression of orotic acid (a metabolite implicated in steatosis in the liver), requires further investigation.

In summary, A. mexicanus troglomorphic populations share many metabolic similarities with human obesity and diabetes mellitus, but also display important differences which may help to explain their resistance to diabetes-like pathologies. We found considerable overlap between the human metabolic signature of obesity and cavefish metabolism. However, we also found important differences in ascorbate, which is known to serve diverse physiological roles, nicotinamide, which is a precursor to $N A D^{+}$synthesis and hence is related to oxidative metabolism, $\alpha$-ketoglutarate, which has been implicated in longevity in C. elegans (18). We have shown via ROS staining that Pachòn cavefish do possess lower levels of superoxide radicals in the liver after 30 days of fasting. This confirms that increased antioxide levels in cavefish do 
indeed correlate with physiological state, and suggests that selection in cave environments favors resistance to oxidative stress. Whether selective pressure is driven chiefly by starvation resistance, or whether it is a mechanism to offset oxidized LDL and other harmful metabolites is an open question.

A. mexicanus cave populations clearly exhibit altered lipoprotein composition and expression of CETP, an important component of lipid homeostasis. Together with increased abundance of antioxidants, this may contribute to the ability of cavefish to withstand high triglyceride levels.

Finally, in order to make our data and methods maximally available to other researchers, we have implemented a transparent pipeline that can be used to regenerate all main figures and tables presented here. We have also used the structured data library ObjTables (53) to provide machine-readable, semantically-accurate representations of the results presented here.

This study provides for the first time (to our knowledge) a large, untargeted metabolomics and lipidomics study of $A$. mexicanus surface and cave morphs. However, there are limitations to our approach. Our analysis was based on juvenile, pre-sexually mature fish. We chose this developmental time point under the hypothesis that evolutionary changes in metabolism would tend to act early to respond to the selective pressure of the cave environment and positive juvenile fish for robust starvation resistance. However, an age of 5 months does not necessarily coincide with seasonally-correlated food shortages, and hence manifestations of starvation adaptations may not occur until a later developmental stage. We also did not examine a fasting duration of greater than 1 month, despite the potential for longer periods of nutrient deprivation in cave environments.

\section{Conclusion}

Our goals for this study were (1) to provide a comprehensive untargeted study of primary metabolites and lipids in A. mexicanus, an extreme-adapted organism with important connections to human health, (2) examine the molecular basis for low-nutrient adaptation in cave-dwelling subpopulations, and (3) identify metabolic changes that might explain A. mexicanus longevity in the face of a phenotype with properties linked to obesity and diabetes.

Our findings show that the adaptation to a low nutrient environment in A. mexicanus is linked to extreme changes in sugar and fat metabolism, and that increased reliance on these energy sources in the liver insulates other tissues from catabolism under long-term fasting.

All in all, our results highlight the role of A. mexicanus as an evolutionary example of extreme metabolism and a model of human obesity, and suggest important roles for certain metabolites in fish and other species.

\section{Acknowledgments}

JKM, JP, TB, LO, RP, JK, SX, and NR were supported by institutional funding from the Stowers Institute for Medi- cal Research. Additionally, NR is supported by the Edward Mallinckrodt Foundation, NIH Grant R01 GM127872, DP2AG071466, NSF IOS-1933428, and EDGE award 1923372.

We are deeply grateful to Zachary Zakibe, Andrew Ingalls, Alba Aparicio Fernandez, David Jewell, and Franchesca Hutton-Lau, Molly Miller for their tireless efforts in maintaining the large fish fish population of the SIMR Cavefish Facility and providing invaluable assistance for this study and others. We would also like to thank Elizabeth Evans, Diana Baumann, M. Shane Merryman, and the SIMR Reptile and Aquatics facility for coordinating and supporting animal research at Stowers and in the Rohner lab in particular, and Rhonda Egidy and Anoja Perera of the Stowers Molecular Biology Facility and Huzaifa Hassan of the Stowers Computational Biology Facility for library construction and analysis of the RNA-Seq study. We would also like to express our sincere thanks to Fengyan Deng, Hannah Wilson, Yongfu Wang, and Cathy McKinney of the Stowers Histology and Microscopy centers resp. for their help in sectioning, staining, and imaging experiments. We are grateful to the West Coast Metabolomics Center for providing data acquisition and analysis for this study.

\section{Competing Interests}

The authors declare no competing interests.

\section{Author Contributions}

JP devised and performed metabolite extraction. JKM performed analysis and wrote the manuscript. TB performed the experiments regarding ROS staining. LO performed caudal vein and lipoprotein experiments. RP contributed sections of the manuscript related to inflammation and immune consequences of antioxidant metabolites. JK performed RNASeq experiments. SX performed validation experiments. NR provided funding, edited the manuscript, and planned experiments.

\section{Bibliography}

1. Ariel C Aspiras, Nicolas Rohner, Brian Martineau, Richard L Borowsky, and Clifford J Tabin. Melanocortin 4 receptor mutations contribute to the adaptation of cavefish to nutrient-poor conditions. Proceedings of the National Academy of Sciences, 112(31):9668-9673, 2015.

2. Shaolei Xiong, Jaya Krishnan, Robert Peuß, and Nicolas Rohner. Early adipogenesis contributes to excess fat accumulation in cave populations of astyanax mexicanus. Developmental biology, 441(2):297-304, 2018.

3. Misty R Riddle, Ariel C Aspiras, Karin Gaudenz, Robert Peuß, Jenny Y Sung, Brian Martineau, Megan Peavey, Andrew C Box, Julius A Tabin, Suzanne McGaugh, et al. Insulin resistance in cavefish as an adaptation to a nutrient-limited environment. Nature, 555(7698): 647-651, 2018.

4. Jaya Krishnan, Jenna L Persons, Robert Peuß, Huzaifa Hassan, Alexander Kenzior, Shaolei Xiong, Luke Olsen, Ernesto Maldonado, Johanna E Kowalko, and Nicolas Rohner. Comparative transcriptome analysis of wild and lab populations of astyanax mexicanus uncovers differential effects of environment and morphotype on gene expression. Journal of Experimental Zoology Part B: Molecular and Developmental Evolution, 2020.

5. Elizabeth T Cirulli, Lining Guo, Christine Leon Swisher, Naisha Shah, Lei Huang, Lori A Napier, Ewen F Kirkness, Tim D Spector, C Thomas Caskey, Bernard Thorens, et al. Profound perturbation of the metabolome in obesity is associated with health risk. Cell metabolism, 29(2):488-500, 2019.

6. Francesco Rubino, David M Nathan, Robert H Eckel, Philip R Schauer, K George MM Alberti, Paul Z Zimmet, Stefano Del Prato, Linong Ji, Shaukat M Sadikot, William H Herman, et al. Metabolic surgery in the treatment algorithm for type 2 diabetes: a joint statement by international diabetes organizations. Surgery for Obesity and Related Diseases, 12(6): 1144-1162, 2016. 
bioRxiv preprint doi: https://doi.org/10.1101/2020.10.27.358077; this version posted July 9, 2021. The copyright holder for this preprint (which was not certified by peer review) is the author/funder, who has granted bioRxiv a license to display the preprint in perpetuity. It is made available under aCC-BY-ND 4.0 International license.

7. Kathrin Hüppop. Oxygen consumption of astyanax fasciatus (characidae, pisces): a comparison of epigean and hypogean populations. Environmental Biology of Fishes, 17(4): 299-308, 1986.

8. Nicola Longo, Yuhuan Wang, and Marzia Pasquali. Progressive decline in insulin levels in rabson-mendenhall syndrome. The Journal of Clinical Endocrinology \& Metabolism, 84(8): 2623-2629, 1999.

9. Jaya Krishnan and Nicolas Rohner. Sweet fish: Fish models for the study of hyperglycemia and diabetes. Journal of diabetes, 11(3):193-203, 2019.

10. Mélanie Viltard, Sylvère Durand, Maria Pérez-Lanzón, Fanny Aprahamian, Deborah Lefevre, Christine Leroy, Frank Madeo, Guido Kroemer, and Gérard Friedlander. The metabolomic signature of extreme longevity: Naked mole rats versus mice. Aging (Albany NY), 11(14):4783, 2019

11. Thomas J Park, Jane Reznick, Bethany L Peterson, Gregory Blass, Damir Omerbašić, Nigel C Bennett, P Henning JL Kuich, Christin Zasada, Brigitte M Browe, Wiebke Hamann, et al. Fructose-driven glycolysis supports anoxia resistance in the naked mole-rat. Science, 356(6335):307-311, 2017.

12. William R Jeffery. Astyanax surface and cave fish morphs. EvoDevo, 11(1):1-10, 2020 .

13. Minoru Kanehisa and Susumu Goto. Kegg: kyoto encyclopedia of genes and genomes. Nucleic acids research, 28(1):27-30, 2000.

14. David S Wishart, Yannick Djoumbou Feunang, Ana Marcu, An Chi Guo, Kevin Liang, Rosa Vázquez-Fresno, Tanvir Sajed, Daniel Johnson, Carin Li, Naama Karu, et al. Hmdb 4.0: the human metabolome database for 2018. Nucleic acids research, 46(D1):D608-D617, 2018.

15. Siming Ma, Sun Hee Yim, Sang-Goo Lee, Eun Bae Kim, Sang-Rae Lee, Kyu-Tae Chang, Rochelle Buffenstein, Kaitlyn N Lewis, Thomas J Park, Richard A Miller, et al. Organization of the mammalian metabolome according to organ function, lineage specialization, and longevity. Cell metabolism, 22(2):332-343, 2015.

16. Johan Trygg and Svante Wold. Orthogonal projections to latent structures (o-pls). Journal of Chemometrics: A Journal of the Chemometrics Society, 16(3):119-128, 2002.

17. Hye-Sook Han, Geon Kang, Jun Seok Kim, Byeong Hoon Choi, and Seung-Hoi Koo. Regulation of glucose metabolism from a liver-centric perspective. Experimental \& molecular medicine, 48(3):e218-e218, 2016

18. Randall M Chin, Xudong Fu, Melody Y Pai, Laurent Vergnes, Heejun Hwang, Gang Deng, Simon Diep, Brett Lomenick, Vijaykumar S Meli, Gabriela C Monsalve, et al. The metabolite $\alpha$-ketoglutarate extends lifespan by inhibiting atp synthase and tor. Nature, 510(7505): 397-401, 2014.

19. Azar Asadi Shahmirzadi, Daniel Edgar, Chen-Yu Liao, Yueh-Mei Hsu, Mark Lucanic, Arash Asadi Shahmirzadi, Christopher D Wiley, Garbo Gan, Dong Eun Kim, Herbert G Kasler, et al. Alpha-ketoglutarate, an endogenous metabolite, extends lifespan and compresses morbidity in aging mice. Cell Metabolism, 32(3):447-456, 2020.

20. John X Wilson. The physiological role of dehydroascorbic acid. FEBS letters, 527(1-3):5-9, 2002.

21. Biyun Ching, Shit $\mathrm{F}$ Chew, and Yuen $\mathrm{K} I \mathrm{I}$. Ascorbate synthesis in fishes: A review. IUBMB life, 67(2):69-76, 2015.

22. Shaohui Huang and Michael P Czech. The glut4 glucose transporter. Cell metabolism, 5 (4):237-252, 2007

23. Javier R Jaldin-Fincati, Martin Pavarotti, Scott Frendo-Cumbo, Philip J Bilan, and Amira Klip. Update on glut4 vesicle traffic: a cornerstone of insulin action. Trends in Endocrinology \& Metabolism, 28(8):597-611, 2017.

24. ES Ford, S Liu, DM Mannino, WH Giles, and SJ Smith. C-reactive protein concentration and concentrations of blood vitamins, carotenoids, and selenium among united states adults. European journal of clinical nutrition, 57(9):1157-1163, 2003.

25. Silvia Isabel Rech Franke, Luiza Louzada Müller, Maria Carolina Santos, Arcênio Fishborn, Liziane Hermes, Patrícia Molz, Camila Schreiner Pereira, Francisca Maria Assmann Wichmann, Jorge André Horta, Sharbel Weidner Maluf, et al. Vitamin c intake reduces the cytotoxicity associated with hyperglycemia in prediabetes and type 2 diabetes. BioMed research international, 2013, 2013.

26. Robert Peuß, Andrew C Box, Shiyuan Chen, Yongfu Wang, Dai Tsuchiya, Jenna L Persons, Alexander Kenzior, Ernesto Maldonado, Jaya Krishnan, Jörn P Scharsack, et al. Adaptation to low parasite abundance affects immune investment and immunopathological responses of cavefish. Nature Ecology \& Evolution, pages 1-15, 2020.

27. Vivian A Paschoal, Evelyn Walenta, Saswata Talukdar, Ariane R Pessentheiner, Olivia Osborn, Nasun Hah, Tyler J Chi, George L Tye, Aaron M Armando, Ronald M Evans, et al. Positive reinforcing mechanisms between gpr120 and ppar $\gamma$ modulate insulin sensitivity. Cell Metabolism, 2020.

28. Christopher $\mathrm{K}$ Glass and Jerrold M Olefsky. Inflammation and lipid signaling in the etiology of insulin resistance. Cell metabolism, 15(5):635-645, 2012.

29. Pauline Morigny, Marianne Houssier, Aline Mairal, Claire Ghilain, Etienne Mouisel, Fadila Benhamed, Bernard Masri, Emeline Recazens, Pierre-Damien Denechaud, Geneviève Tavernier, et al. Interaction between hormone-sensitive lipase and chrebp in fat cells controls insulin sensitivity. Nature Metabolism, 1(1):133-146, 2019.

30. Fredrik Karpe, Julian R Dickmann, and Keith N Frayn. Fatty acids, obesity, and insulin resistance: time for a reevaluation. Diabetes, 60(10):2441-2449, 2011.

31. David Young Oh, Saswata Talukdar, Eun Ju Bae, Takeshi Imamura, Hidetaka Morinaga, WuQiang Fan, Pingping Li, Wendell J Lu, Steven M Watkins, Jerrold M Olefsky, et al. Gpr120 is an omega-3 fatty acid receptor mediating potent anti-inflammatory and insulinsensitizing effects. Cell, 142(5):687-698, 2010.

32. Takayuki Teruya, Romanas Chaleckis, Junko Takada, Mitsuhiro Yanagida, and Hiroshi Kondoh. Diverse metabolic reactions activated during 58 -hr fasting are revealed by non-targeted metabolomic analysis of human blood. Scientific reports, 9(1):1-11, 2019.

33. Jaya Krishnan, Chris Seidel, Ning Zhang, Jake VanCampen, Robert Peuss, Shaolei Xiong, Alexander Kenzior, Hua Li, Joan W Conaway, and Nicolas Rohner. Genome-wide analysis of cis-regulatory changes in the metabolic adaptation of cavefish. bioRxiv, 2020.

34. Nir Barzilai, Gil Atzmon, Clyde Schechter, Ernst J Schaefer, Adrienne L Cupples, Richard Lipton, Suzanne Cheng, and Alan R Shuldiner. Unique lipoprotein phenotype and genotype associated with exceptional longevity. Jama, 290(15):2030-2040, 2003.

35. JK Christison, Kerry-Anne Rye, and Roland Stocker. Exchange of oxidized cholesteryl linoleate between Idl and hdl mediated by cholesteryl ester transfer protein. Journal of lipid research, 36(9):2017-2026, 1995.

36. Margaret E Brousseau, Ernst J Schaefer, Megan L Wolfe, LeAnne T Bloedon, Andres G Digenio, Ronald W Clark, James P Mancuso, and Daniel J Rader. Effects of an inhibitor of cholesteryl ester transfer protein on hdl cholesterol. New England Journal of Medicine, 350 (15):1505-1515, 2004

37. Shobha Ghosh, Bin Zhao, Jinghua Bie, and Jingmei Song. Macrophage cholesteryl ester mobilization and atherosclerosis. Vascular pharmacology, 52(1-2):1-10, 2010.

38. Xiao-Hua Yu, Yu-Chang Fu, Da-Wei Zhang, Kai Yin, and Chao-Ke Tang. Foam cells in atherosclerosis. Clinica chimica acta, 424:245-252, 2013.

39. Philip J Barter and Kerry-Anne Rye. Cholesteryl ester transfer protein inhibition as a strategy to reduce cardiovascular risk. Journal of lipid research, 53(9):1755-1766, 2012

40. Longhou Fang, Chao Liu, and Yury I Miller. Zebrafish models of dyslipidemia: relevance to atherosclerosis and angiogenesis. Translational Research, 163(2):99-108, 2014.

41. Daniel Steinberg. Antioxidants and atherosclerosis. a current assessment. Circulation, 84 (3):1420-1425, 1991

42. Sona Mitra, Tanu Goyal, and Jawahar L Mehta. Oxidized Idl, lox-1 and atherosclerosis. Cardiovascular Drugs and Therapy, 25(5):419, 2011

43. Konstantin Stoletov, Longhou Fang, Soo-Ho Choi, Karsten Hartvigsen, Lotte F Hansen, Chris Hall, Jennifer Pattison, Joseph Juliano, Elizabeth R Miller, Felicidad Almazan, et al. Vascular lipid accumulation, lipoprotein oxidation, and macrophage lipid uptake in hypercholesterolemic zebrafish. Circulation research, 104(8):952-960, 2009.

44. Rafael A Cox and Mario R García-Palmieri. Cholesterol, triglycerides, and associated lipoproteins. 2011.

45. Mason W. Freeman and Geoffrey A. Walford. Chapter 41 - lipoprotein metabolism and the treatment of lipid disorders. In J. Larry Jameson, Leslie J De Groot, David M. de Kretser, Linda C. Giudice, Ashley B. Grossman, Shlomo Melmed, John T. Potts, and Gordon C. Weir, editors, Endocrinology: Adult and Pediatric (Seventh Edition), pages 715-736.e7. W.B. Saunders, Philadelphia, seventh edition edition, 2016. ISBN 978-0-323-18907-1. doi: https://doi.org/10.1016/B978-0-323-18907-1.00041-X.

46. Andrew Gelman, Aleks Jakulin, Maria Grazia Pittau, Yu-Sung Su, et al. A weakly informative default prior distribution for logistic and other regression models. The annals of applied statistics, 2(4):1360-1383, 2008.

47. Susan B Standerfer and Philip Handler. Fatty liver induced by orotic acid feeding. Proceedings of the Society for Experimental Biology and Medicine, 90(1):270-271, 1955.

48. Seung-Hoi Koo. Nonalcoholic fatty liver disease: molecular mechanisms for the hepatic steatosis. Clinical and molecular hepatology, 19(3):210, 2013.

49. Kerry L Donnelly, Coleman I Smith, Sarah J Schwarzenberg, Jose Jessurun, Mark D Boldt, Elizabeth J Parks, et al. Sources of fatty acids stored in liver and secreted via lipoproteins in patients with nonalcoholic fatty liver disease. The Journal of clinical investigation, 115(5): 1343-1351, 2005.

50. Miriam Furne and Ana Sanz. Starvation in fish - sturgeon and rainbow trout as examples. In V. R. Preedy and V. B. Patel, editors, Handbook of Famine, Starvation, and Nutrient Deprivation. Springer International Publishing AG, 2017.

51. Alfonso Pompella, Athanase Visvikis, Aldo Paolicchi, Vincenzo De Tata, and Alessandro F Casini. The changing faces of glutathione, a cellular protagonist. Biochemical pharmacology, 66(8):1499-1503, 2003

52. Alexandra Vaccaro, Yosef Kaplan Dor, Keishi Nambara, Elizabeth A Pollina, Cindy Lin, Michael E Greenberg, and Dragana Rogulja. Sleep loss can cause death through accumulation of reactive oxygen species in the gut. Cell, 181(6):1307-1328, 2020.

53. Jonathan R Karr, Wolfram Liebermeister, Arthur P Goldberg, John AP Sekar, and Bilal Shaikh. Structured spreadsheets with objtables enable data reuse and integration. arXiv preprint arXiv:2005.05227, 2020.

54. Vitali Matyash, Gerhard Liebisch, Teymuras V Kurzchalia, Andrej Shevchenko, and Dominik Schwudke. Lipid extraction by methyl-tert-butyl ether for high-throughput lipidomics. Journal of lipid research, 49(5):1137-1146, 2008.

55. Oliver Fiehn. Metabolomics by gas chromatography-mass spectrometry: Combined targeted and untargeted profiling. Current protocols in molecular biology, 114(1):30-4, 2016.

56. Tomas Cajka and Oliver Fiehn. Comprehensive analysis of lipids in biological systems by liquid chromatography-mass spectrometry. TrAC Trends in Analytical Chemistry, 61:192206, 2014.

57. Oliver Fiehn, Gert Wohlgemuth, Martin Scholz, Tobias Kind, Do Yup Lee, Yun Lu, Stephanie Moon, and Basil Nikolau. Quality control for plant metabolomics: reporting msi-compliant studies. The Plant Journal, 53(4):691-704, 2008

58. Kirsten Skogerson, Gert Wohlgemuth, Dinesh K Barupal, and Oliver Fiehn. The volatile compound binbase mass spectral database. BMC bioinformatics, 12(1):321, 2011.

59. Hiroshi Tsugawa, Tomas Cajka, Tobias Kind, Yan Ma, Brendan Higgins, Kazutaka Ikeda, Mitsuhiro Kanazawa, Jean VanderGheynst, Oliver Fiehn, and Masanori Arita. Ms-dial: data-independent $\mathrm{ms} / \mathrm{ms}$ deconvolution for comprehensive metabolome analysis. Nature methods, 12(6):523-526, 2015.

60. Brian C DeFelice, Sajian Singh Mehta, Stephanie Samra, Tomas Cajka, Benjamin Wancewicz, Johannes F Fahrmann, and Oliver Fiehn. Mass spectral feature list optimizer (ms-flo): a tool to minimize false positive peak reports in untargeted liquid chromatographymass spectroscopy (Ic-ms) data processing. Analytical chemistry, 89(6):3250-3255, 2017.

61. Tobias Kind, Kwang-Hyeon Liu, Do Yup Lee, Brian DeFelice, John K Meissen, and Oliver Fiehn. Lipidblast in silico tandem mass spectrometry database for lipid identification. Nature methods, 10(8):755-758, 2013.

62. TS Imam, U Bala, ML Balarabe, TI Oyeyi, et al. Length-weight relationship and condition factor of four fish species from wasai reservoir in kano, nigeria. African Journal of General Agriculture, 6(3):125-130, 2010

63. Johan A Westerhuis, Ewoud JJ van Velzen, Huub CJ Hoefsloot, and Age K Smilde. Discriminant q 2 (dq 2) for improved discrimination in plsda models. Metabolomics, 4(4):293-296, 2008.

64. Dongming Huang, Nathan Stein, Donald B Rubin, and SC Kou. Catalytic prior distributions with application to generalized linear models. Proceedings of the National Academy of Sciences, 117(22):12004-12010, 2020. 


\section{Materials and Methods}

\section{Experimental Model and Subject Details}

Surface morphs of Astyanax mexicanus were reared from offspring of Mexican surface fish collected in the Río Choy. Pachón and Tinaja morphs were reared from fish originating from the Pachón and Tinaja caves. A total of 18 fish from each population were used in experiments. Sex was not determined due to difficulties in determining sex in juvenile A. mexicanus fish. This study was approved by the Institutional Animal Care and Use Committee (IACUC) of the Stowers Institute for Medical Research under protocol 2019084. Animals were euthanized according to an IACUCapproved euthanasia protocols based on American Veterinary Medical Association (AVMA) guidelines using Tricaine mesylate. The method currently in use has been updated to reflect 2020 AVMA guidelines and uses 30 minutes of opercular movement cessation unless a secondary method is employed.

\section{Method Details}

Fish husbandry All Astyanax are housed in glass fish tanks on recirculating aquaculture racks (Pentair, Apopka, FL) with a 14:10 LD photoperiod. Each rack system is equipped with mechanical, chemical and biological filtration and UV disinfection. Water quality parameters are monitored and maintained daily as described in previous studies $(2,26)$. Fish were fed once per day with mysis shrimp and twice per day with Gemma diet. Gemma feed is Protein 59\%; Lipids 14\%; Fiber 0.2\%; Ash 14\%; Phosphorus 1.3\%; Calcium 1.5\%; Sodium 0.7\%; Vitamin A $23000 \mathrm{IU} / \mathrm{kg}$; Vitamin D3 $2800 \mathrm{IU} / \mathrm{kg}$; Vitamin C $1000 \mathrm{mg} / \mathrm{kg}$; Vitamin E $400 \mathrm{mg} / \mathrm{kg}$. Health examinations of all fish were conducted by aquatics staff twice daily. Astyanax colonies are screened biannually for ectoparasites and endoparasites and no pathogens were present at the time of this study. Fish treatment and care was approved by the Institutional Animal Care and Use Committee (IACUC) of the Stowers Institute for Medical Research. NR's institutional authorization for use of Astyanax mexicanus in research is 2019-084.

Feeding regimen and tissue collection Age-matched offspring of Surface, Pachón, and Tinaja populations were reared in similar densities at $23^{\circ} \mathrm{C}$ in $14: 10 \mathrm{LD}$ cycles as described previously. Fish were the result of a group mating event within populations. Fish were housed only with members of their population for their entire lives. At 4-months (Tinaja), 4 months and 1 day (Pachón), 4 months and 2 days (Surface), fish of each population were separated into two tanks. 12 fish were separated and starved for 30-days until tissue collection. The 12 fish were maintained on regular feeding schedules until 4-days prior to tissue collection when food was withheld from each population's regular feeding tank. The mass $(\mathrm{g})$ and length $(\mathrm{cm})$ of each fish was recorded at separation. All efforts were made to equalize mass and length distributions in each cohort. On the evening before tissue collection, 6 fish from the 4-day starved tank were separated and placed into three, 3L-tanks. Tanks were divided down the middle such that all 6 fish (2 in each tank) were housed individually. Singly housed fish were refed for 3-hours with 10mg of Gemma 500 on the morning of the dissection day for each population. Dissection occurred at 5 months (October 5th, 2019; Tinaja), 5 months and 1 day (October 6th, 2019; Pachón), 5 months and 2 days (October 7th, 2019; Surface). Fish were re-fed in intervals between 8:30am-12:00pm. At each 3-hour time point, a re-fed fish, a 4-day starved fish, and a 30-day starved fish was euthanized in MS-222. To reduce variability between populations dissected on subsequent days, all dissections took place between 11:30-3pm and were handled identically. Prior to dissection, the final mass and length were recorded for each fish. The liver, muscle, and brain were dissected and placed in $1.5 \mathrm{~mL}$ plastic tubes. Tissues were flash frozen on liquid nitrogen, transferred to dry ice and stored at $-80 \mathrm{C}$. Samples were shipped to West Coast Metabolomics Center on dry ice overnight for analysis.

Sample Preparation Samples were prepared using the Matyash protocol (54). This procedure allows efficient extraction of lipids in a non-polar methyl tert-butyl ether (MTBE) layer, and extraction of primary metabolites in the polar water/methanol layer $(55,56)$. From each sample, 4.1 $\mathrm{mg}$ of frozen liver or brain tissue $(+/-0.3 \mathrm{mg})$ or $10.1 \mathrm{mg}$ of muscle tissues $(+/-0.3 \mathrm{mg})$ was weighed and placed into 1.5 $\mathrm{mL}$ Eppendorf tubes. Samples were ground prior to extraction using beads with a Spex Sample Prep GenoGrinder with stainless steel $2-3 \mathrm{~mm}$ beads for $30 \mathrm{~s}$. $975 \mu \mathrm{L}$ of ice cold, 3:10 (v/v) MeOH/MTBE + QC mix/CE (22:1) extraction solvent was added to each homogenized sample. Samples were vortexed for $10 \mathrm{sec}$ and shaken for $5 \mathrm{~min}$ at $4^{\circ} \mathrm{C} .188 \mathrm{uL}$ room temperature LC/MS water was added and samples vortexed for $20 \mathrm{sec}$, then centrifuged for $2 \mathrm{~min}$ at 14,000 rcf. The upper organic phase was transferred to two separate tubes (350 $\mu \mathrm{L}$ each) for lipidomics (CSH) analysis. The bottom aqueous phase was transferred to two additional tubes $(110 \mu \mathrm{L}$ each) for primary metabolism (GC-TOF) analysis. One tube from each phase was reserved as a backup, the other tube was dried down completely using centrivap. Both were kept at $20^{\circ} \mathrm{C}$ until ready for analysis. As an additional step prior to GC-TOF analysis, samples were resuspended in $500 \mu \mathrm{L}$ of degassed, $-20^{\circ} \mathrm{C}$ mixture of acetonitrile (ACN): isopropanol (IPA): water $(\mathrm{H} 2 \mathrm{O})(3: 3: 2, \mathrm{v} / \mathrm{v} / \mathrm{v})$. Samples were vortexed for $10 \mathrm{sec}$ and then centrifuged at 14,000 $\mathrm{rcf}$ for $2 \mathrm{~min}$. 450 $\mu \mathrm{L}$ of supernatant was transferred to a new tube and concentrated to complete dryness using a Labconco Centruvap cold concentrator.

Primary Metabolite Data Acquisition Metabolite abundances were quantified by gas-chromatography, time-offlight mass spectrometry (GC-TOF/MS) using previously established methods (57). Briefly, an Agilent 6890 gas chromatograph (Santa Clara, CA) equipped with a Gerstel automatic linear exchange systems (ALEX) which included a multipurpose sample dual rail and a Gerstel cold injection system (CIS) was used with a Leco Pagasus IV time-of-flight 
mass spectrometer running Leco ChromaTOF software. The injection temperature was ramped from $50^{\circ} \mathrm{C}$ to a final temperature of $275^{\circ} \mathrm{C}$ at a rate of $12^{\circ} \mathrm{C} / \mathrm{s}$ and held for 3 minutes. Injection volume was $0.5 \mu \mathrm{l}$ with $10 \mu \mathrm{l} / \mathrm{s}$ injection speed on a splitless injector with a purge time of 25 seconds. The liner (Gerstel \# 011711-010-00) was changed automatically every 10 samples to reduce sample carryover. The injection syringe was washed three times with $10 \mu \mathrm{L}$ ethyl acetate before and after each injection. For gas chromatography, a Rtx-5Sil MS column (30 m long, $0.25 \mathrm{~mm}$ i.d.) with $0.25 \mu \mathrm{m} 95 \%$ dimethyl 5\% diphenyl polysiloxane film was used (Restek, Bellefonte PA). The GC column was equipped with an additional $10 \mathrm{~m}$ integrated guard column. $99.9999 \%$ pure Helium with a built-in purifier was set at a flow rate of $1 \mathrm{~mL} /$ minute. The oven temperature was held constant at $50^{\circ} \mathrm{C}$ for 1 minute, ramped at $20^{\circ} \mathrm{C} /$ minute to $330^{\circ} \mathrm{C}$, and then held constant for 5 minutes. The transfer line temperature between gas chromatograph and mass spectrometer was set to $280^{\circ} \mathrm{C}$. The mass spectra were acquired at a rate of 17 spectra/second, with a scan mass range of 80-500 Da at an ionization energy of $-70 \mathrm{eV}, 1800 \mathrm{~V}$ detector voltage, and $250^{\circ} \mathrm{C}$ ion source.

Primary Metabolite Data Processing Raw GC-TOF MS data files were preprocessed using ChromaTOF version 4.0 without smoothing, a $3 \mathrm{~s}$ peak width, baseline subtraction just above the noise level, and automatic mass spectral deconvolution and peak detection at signal/noise $(\mathrm{s} / \mathrm{n})$ levels of 5:1 throughout the chromatogram. Results were exported with absolute spectra intensities and further processed by a filtering algorithm implemented in the metabolomics BinBase database (58). The BinBase algorithm ( $r t x 5)$ used the following settings: validity of chromatogram (107 counts/s), unbiased retention index marker detection (MS similarity $>$ 800 , validity of intensity range for high $\mathrm{m} / \mathrm{z}$ marker ions), retention index calculation by 5 th order polynomial regression. Spectra were cut to 5\% base peak abundance and matched to database entries from most to least abundant spectra using the following matching filters: retention index window \pm 2000 units (equivalent to about $\pm 2 \mathrm{~s}$ retention time), validation of unique ions and apex masses (unique ion must be included in apexing masses and present at $>3 \%$ of base peak abundance), mass spectrum similarity must fit criteria dependent on peak purity and signal/noise ratios and a final isomer filter. Failed spectra were automatically entered as new database entries if signal/noise ratios were larger than 25 and mass spectral purity better than $80 \%$. Data was reported as peak height using the unique quantification ion at the specific retention index, unless a different quantification ion was manually set in the BinBase administration software BinView.

Lipid Data Acquisition Lipid abundances were determined by charged-surface hybrid column-electrospray ionization quadrupole time-of-flight tandem mass spectrometry (CSHESI QTOF MS/MS). For positively charged lipids, an Agilent 6530 QTOF mass spectrometer with resolution 10,000 was used and for negatively charged lipids, an Agilent 6550 QTOF mass spectrometer with resolution 20,000 was used. Electrospray ionization was used to ionize col- umn elutants in both positive and negative modes. Compounds were separated using a Waters Acquity ultra-highpressure, liquid-chromatography charged surface hybrid column (UPLC CSH) C18 (100 mm length $\times 2.1 \mathrm{~mm}$ internal diameter; 1.7 um particles). The conditions in positive mode were as follows: mobile phase A (60:40 acetonitrile:water $+10 \mathrm{mM}$ ammonium formiate $+0.1 \%$ formic acid, mobile phase B (90:10 isopropanol:acetonitrile $+10 \mathrm{mM}$ ammonium formiate $+0.1 \%$ formic acid). The conditions in negative mode were as follows: mobile phase A (60:40 acetonitrile:water $+10 \mathrm{mM}$ ammonium acetate), mobile phase B (90:10 isopropanol:acetonitrile $+10 \mathrm{mM}$ ammonium acetate). $5 \mu \mathrm{L}$ of each brain, liver and muscle sample was injected in negative mode. $0.5 \mathrm{uL}$ of each brain and liver, and $0.25 \mathrm{uL}$ of muscle samples was injected in positive mode. In both modes, the column temperature was $65^{\circ} \mathrm{C}$, at a flow rate of $0.6 \mathrm{~mL} /$ minute, an injection temperature of $4{ }^{\circ} \mathrm{C}$, and a gradient of 0 minutes $15 \%, 0-2$ minutes $30 \%, 2-2.5$ minutes $48 \%, 2.5-11$ minutes $82 \%, 11-11.5$ minutes $99 \%, 11.5-12$ minutes $99 \%, 12-12.1$ minutes $15 \%$, and $12.1-15$ minutes $15 \%$. The ESI capillary voltage was set to +3.5 and -3.5 $\mathrm{kV}$, and the collision energy to 25 for positive and negative modes. Mass-to-charge ratios $(\mathrm{m} / \mathrm{z})$ were scanned from 60 to $1200 \mathrm{Da}$ and spectra acquired every 2 seconds. Automatic valve switching was used after each injection to reduce sample carryover for highly lipophilic compounds.

A Lipid Data Processing Raw lipidomic data were processed using MS-DIAL (59) followed by blank subtractions in Microsoft Excel and data cleanup using MS-FLO (60). Briefly, data were converted to files using Abf Converter. All default parameters were used for processing of MS-DIAL data, except for minimum peak height and width which were adjusted to the instrument. Results are exported from MSDIAL and a blank reduction is performed for all features which are found in at least one sample. Blank reduction takes the maximum peak height relative to the blank average height and the average of all non-zero peak heights for samples. Duplicates and isotopes are examined using MS-FLO and deleted if confirmed. Peaks were annotated by manually comparing the MS/MS spectra and the accurate masses of precursor ions to spectra in the Fiehn laboratory LipidBlast spectral library (61). Additional peaks are manually curated from sample chromatograms. Manually curation was confirmed by using MassHunter Quant software to verify peak candidates based on peak shape and height reproducibility, and retention time reproducibility in replicate samples. The data were reported as peak heights for the specific quantification ion at the specific retention time.

\section{Quantification and Statistical Analysis}

A.1 Weight change and K-factor calculations Percent weight change for each fish was calculated using formula 1. Mass and length measurements were recorded at the beginning and end of feeding regimens.

$$
\Delta W t(\%)=\left(m_{\text {final }}-m_{\text {initial }}\right) / m_{\text {final }} \times 100,
$$


where $m$ is mass. $\mathrm{K}$-factor is a metric that represents both the mass and length of individuals and is frequently used in aquaculture research to assess an animal's physical condition (62). K-factor for each fish was calculated at the beginning of feeding regimens (app. 4 months) and on the day of dissection (30-days later) using the formula (c) below. Percent $\mathrm{K}$-factor change was calculated using formula (d).

$$
K=\left(m / x^{3}\right) \times 100,
$$

where $x$ is the standard length.

$$
\Delta K(\%)=\left(K_{\text {final }}-K_{\text {initial }}\right) / K_{\text {final }} \times 100
$$

Data in Supplemental Fig S2 and Table S1 were analyzed and graphically represented using Prism software (GraphPad, Prism version 8.3.0 for Mac, GraphPad Software, San Diego, California USA). Data was first analyzed for normality using four independent methods: D'Agostino-Pearson, ShapiroWilk, Kolmogorov-Smirnov, Anderson-Darling. When comparing between more than two groups, data that passed three of four normality tests were analyzed using One-way ANOVA with Tukey correction for multiple comparisons between all groups. Data which failed more than one normality test, was analyzed with Kruskal-Wallis test using Dunn's for multiple comparison correction. The tests used in each figure are reported in the figure legends. p-values less than 0.05 are reported and the level of significance is indicated using the ${ }^{*}$ system (ns, $p>0.05 ; *, p \leqslant 0.05 ; * *, p \leqslant 0.01 ; * * *$, $p \leqslant 0.001 ; * * * *, p \leqslant 0.0001)$.

Further Data Processing Processed primary metabolite data were vector normalized using mTIC. First, the sum of all peak heights for all identified metabolites, excluding the unknowns, for each sample was calculated. Such peak sums are called "mTIC" and represent the sum of genuine metabolites (identified compounds) in each sample. This method avoids unidentified peaks that could represent potentially non-biological artifacts such as column bleed, contaminants, or routine machine maintenance. mTIC averages for each sample were compared to determine if the variance between samples was significantly different $(\mathrm{p}<0.05)$. Samples were then normalized to the average mTIC "mTICaverage" within populations (Surface, Pachón, or Tinaja) and within organs (brain, muscle, or liver). For example, each biological replicate of the Tinaja brain group was normalized to the average mTIC of all Tinaja brain replicates regardless of feeding state. The equation (a) below was then used to normalize each metabolite (i) of a sample (j). After normalization, data are reported as 'relative semi-quantifications' or normalized peak heights.

$$
y_{i j}(\text { normalized })=\left(x_{i j, \text { raw }} / \mathrm{mTIC}_{j}\right) \times \overline{\text { mTIC }}
$$

where $x_{i j}$ is the raw peak intensity for metabolite $i$ in sample $j, \mathrm{mTIC}_{j}$ is the average identified peak intensity in sample $j$, $\overline{\mathrm{mTIC}}$ is the global average identified peak intensity, and $y_{i j}$ is the mTIC-normalized intensity of metabolite $i$ in sample $j$.
Metabolite Categorization Metabolites were categorized according to their respective subclass classification in the human metabolite database (14) (if the subclass was absent, we instead used the superclass of the respective metabolite). Metabolite classes with low membership were manually reassigned to arrive at five broad metabolite categories:

- Carbohydrates and central carbon metabolites (CCM). Simple sugars such as glucose, fructose, and various phosphates thereof, as well as core metabolites in glycolysis, gluconeogenesis, the TCA cycle, and the pentose phosphate pathway.

- Amino acids. All amino acids and intermediates in amino acid biosynthesis and degradation.

- Fatty acids. All free fatty acids, intermediates, and metabolites involved in lipogenesis and $\beta$-oxidation.

- Miscellaneous / secondary metabolites. Metabolites that do not fall in any of the other categories.

- Nucleotides. All nucleotides, nucleosides, nucleobases, and byproducts / intermediates of nucleotide metabolism.

Within each of metabolite category, we further normalized $\log _{10}$ peak intensities using $\mathrm{z}$-score normalization prior to performing PCA, O-PLS (described below) or any supervised classification or statistical modeling.

O-PLS In order to remove sources of variation not useful in discriminating the feeding state of different samples, we used O-PLS (16), a technique commonly used in spectroscopy to correct for systematic variation (16). O-PLS is often applied to raw spectra in order to eliminate the influence of background signals, but here we apply it instead to mTIC normalized peak intensities. Our main use of O-PLS is to remove biological noise that is uncorrelated with feeding state, such as baseline differences or trends among different populations. While z-score normalization already removes many of these artifacts, we observed that O-PLS generally enhanced the predictive accuracy of our PLS classifier. Given an input matrix $X$ of $n$ samples and $m$ spectral features (metabolite peak intensities in our study), and a target matrix $Y$ of classes or measured values (here, the feeding state), the final output of O-PLS (referred to here as $X^{\prime}$ ) is again an $m$ by $n$ matrix consisting of $X$ with the systematic variation orthogonal to $Y$ removed.

Characterization of Feeding State Responses In order to determine which tissues and metabolite categories are most strongly implicated in (1) the starvation response within a given population, (2) differences in metabolite levels between different populations for a given feeding state, we used a simple 1-component PLS classifier trained on the output $X^{\prime}$ of O-PLS.

The discriminant $\mathrm{Q}^{2}$ value is a metric of PLS model accuracy and is given by 


$$
\mathrm{Q}^{2}=1-\frac{\sum_{k}\left(y_{k}-\hat{y}_{k}\right)^{2}}{\sum_{k}\left(y_{k}-\hat{y}_{k}\right)^{2}}
$$

However, we use a truncated version $\mathrm{DQ}^{2}(63)$, where $y_{k}$ is replaced by $y_{k}^{\prime}=\max \left(\min \left(y_{k}, 1\right),-1\right)$ and $y_{k}^{\prime}$ is used in place of $y_{k}$. This metric does not penalize the PLS model for correct predictions that overshoot the target class label.

Using this framework, we employed a two-step model comprised of a O-PLS model followed by a single-component PLS model to discriminate refed versus long term-starved samples. We trained this combined model on $\mathrm{z}$ scorenormalized log-transformed data for primary metabolites subdivided into categories. The output of the initial-stage O-PLS model consists of the original data with a PLS component representing 'orthogonal' noise removed. This denoised data was then used to train a one-component PLS classifier on labels representing feeding state. This results in a $\mathrm{DQ}^{2}$ value for the ability to discriminate refed versus starved states. Finally, an iterative scheme was used to randomly permute the label indices of the input data, resulting in a distribution of $\mathrm{DQ}^{2}$ values. The significance level of the original predictive $\mathrm{DQ}^{2}$ value was calculated using a twotailed survival function of a normal distribution fitted to the $\mathrm{DQ}^{2}$ values.

Identification of Significant Metabolites We employed a logistic regression model to identify important features (metabolites, lipids and classes thereof). We were specifically interested in marginal p-values of each individual metabolite, hence we constructed separate single-covariate models for each metabolite or lipid. Models were further based on different types of comparisons: (1) we compared different feeding states within a given population and (2) different populations within a given feeding state. Logistic regression models (and GLMs in general) tend to suffer from complete separation of observed covariates (64). This renders maximum-likelihood estimates of the model parameters impossible. We therefore used the bayesglm function of the arm R package (46) to obtain estimates for model coefficients, even in the case of complete separation. The bayesglm requires specifying a prior distribution. We found that the highly conservative default prior (corresponding to a an assumption that the response to a change in input should typically not exceed roughly \pm 5 on the logistic scale, or, equivalently, no typical change in input should cause a shift in probability from 0.01 to 0.50 , or 0.50 to $0.99(46)$ ) was sufficient to identify important metabolic changes in our comparisons. However, given the conservative nature of this prior distribution, we did not perform FDR correction.

Fig S1 shows the procedure for training the GLM. We first split the input dataset into two matrices: one containing populations as category labels (bottom left), and one containing feeding states as category labels (upper right). We then subset each of these into the three possible pairwise comparisons from each group, compute z-score-normalized values within the comparison, filter the resulting matrix using O-PLS to remove orthogonal noise, and use the bayesglm function to fit a model to the respective comparison for discriminating either populations (within a given feeding state) or feeding states (within a given population). In each case, the GLM consists of a single covariant corresponding to metabolite / lipid peak heights (for individual metabolites) or classes of metabolites / lipids (for Table S4 and S3).

\section{Differences between Feeding States and Shared Metabolites}

To identify metabolites that might play a role in cave adaptation, we sought to fit logistic regression to an input capturing the difference between refed and and starved samples and differences between cave and surface populations simultaneous. We implemented this using the following formula:

$$
x=(P+T) / 2-S,
$$

where $P, T$, and $S$ are z-score normalized mTIC peak intensities for starved vs. refed samples. In general, these vectors have length 12 ( 6 refed and 6 starved samples). A Bayesian logistic regression model was then fitted to the $x$ vector for each metabolite as before, with each element of the response vector labelled accordingly (starved or refed).

ROS Staining and Microscopy We repeated the original starvation experiment using 12 fish each from Pachòn and surface populations. The age of fish was $138 \mathrm{dpf}$ at the time of collection. Following the original fasting procedure, six fish from each population were separated and fasted for 30 days. The remaining six fish in each population were fasted for 4 days and refed for at least three hours on the morning of collection. However, three fish from the surface 30-day fasted group did not survive until collection and were not used in the experiment.

Pachòn and surface fish were dissected alternately. Dissections were split across two days, with 3 fish each in all four experimental groups on the first day and 3 fish each in all groups except 30-day fasted surface on the second day. Livers were sectioned on $4 \%$ agarose gel and each section was stained with a primary dye and DAPI. Primary dyes included dihydroethidium (DHE), MitoSox, and MitoTracker. Sections were imaged with a ZEISS LSM 780 Laser Scanning Microscope. A Z-stack was taken at a location with suitable cell density for each sample.

Quantitation for cytoplasmic and nuclear intensity was accomplished by first taking a 2D slice from the middle plane. We created a threshold mask for the dye signal and DAPI using the average intensity of all Z-slices (not only the middle slice) and intensity measured on the middle plane to compute total intensity. We then took either the difference or intersection of the signal (DHE, MitoSox) and nuclear stain (DAPI) masks depending on whether cytoplasmic or nuclear signal was desired. We then integrated the signal and using the Kruskal-Wallis test to determine significance.

Caudal Vein Imaging Tail sections were taken from 3 fish each of adult Pachòn and surface populations. Sections were stained using Van Gieson staining because of its reported ability to detect fatty streaks in zebrafish larvae (43). 
bioRxiv preprint doi: https://doi.org/10.1101/2020.10.27.358077; this version posted July 9, 2021. The copyright holder for this preprint (which was not certified by peer review) is the author/funder, who has granted bioRxiv a license to display the preprint in perpetuity. It is made

Lipoprotein Measurement and Quantitation Absolute HDL an LDL/VLDL were quantified in Pachon and surface using a Sigma-Aldrich HDL and LDL/VLDL quantitation kit (MAK045). Blood was collected from the caudal vein, spundown, and serum fraction was extracted and diluted 50-fold. Lipoprotein concentration was calculated using OLS curve fitting based on blanks and cholesterol standard measurements at known concentrations (see Table S12 for standard concentrations) and by using $50 \times 2=100$-fold dilution factor as samples were additionally diluted 2-fold in precipitation buffer in accordance with usage instructions supplied with the kit. Three biological replicates were used for each population (Pachon / surface), and two technical replicates were taken for each biological replicate and averaged to calculate the final result for each biological replicate. Error bars represent $95 \%$ confidence intervals for the three biological replicates in each population (where each value is the average of two technical replicates). The HDL/LDL ratio was calculated by dividing raw colorimetric readings for HDL and LDL/VLDL in order to avoid potential bias from OLS. Absolute difference of HDL concentration between Pachòn and surface was statistically significant by a Kruskall-Wallis test, but all other groups were non-significant.

\section{Additional Resources}

The Shiny App for this study may be accessed at https: //cavefin.shinyapps.io/shiny. 


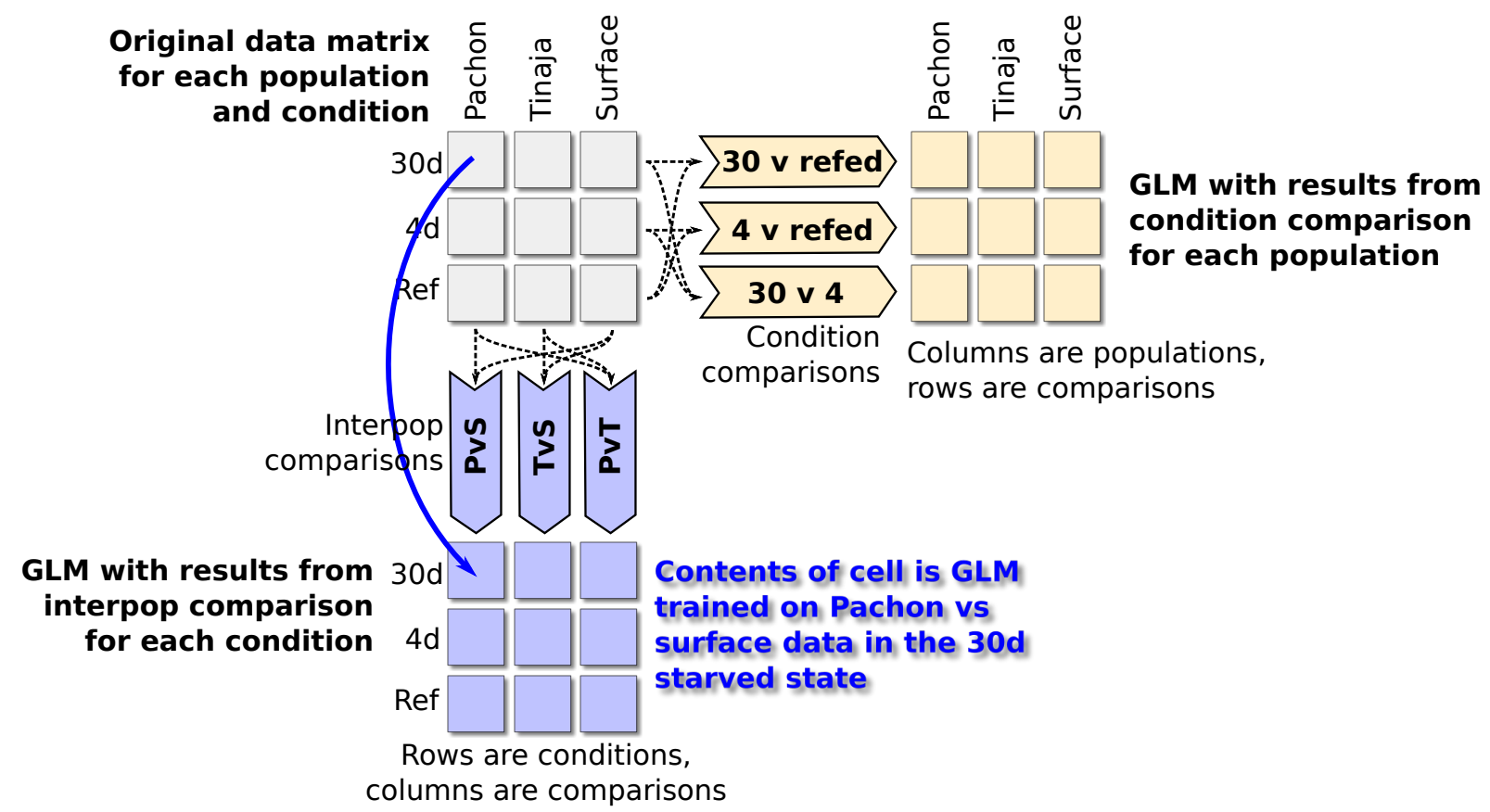

Fig. S1. Schematic depiction of comparisons used for fitting GLM parameters. A Bayesian logistic regression model was used to identify significant features (metabolites and categories) among different groups. To train the model, we first split the entire dataset (upper-left matrix) into two components of pairwise comparisons: a matrix containing category labels for every pairwise comparison of the three populations in our study (lower-left, for example the PvS comparison contains category labels for Pachón and surface and excludes data from Tinaja), and a separate matrix containing category labels for every pairwise comparison of feeding conditions. We then trained a Bayesian logistic regression model using the bayesg $\mathrm{lm} R$ package using the appropriate category labels for each comparison. Predictors were either O-PLS-filtered metabolite peaks or metabolite classes (e.g. fatty acids, nucleotides, etc.).

A

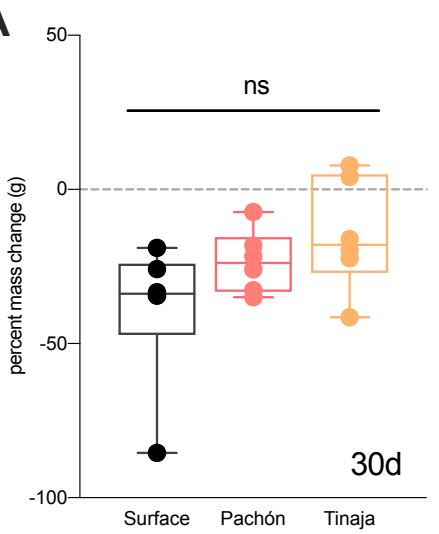

D

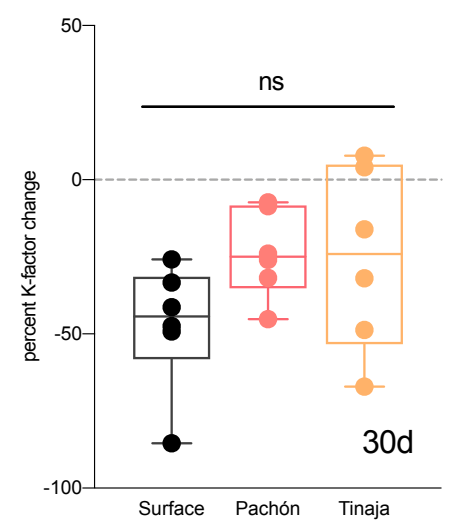

B

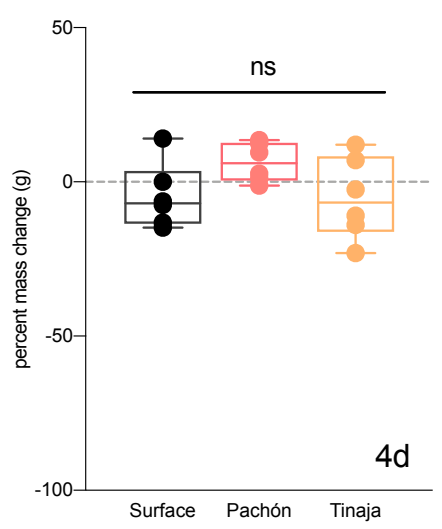

E

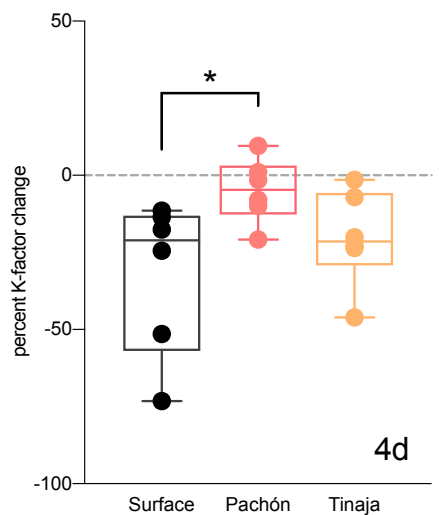

C

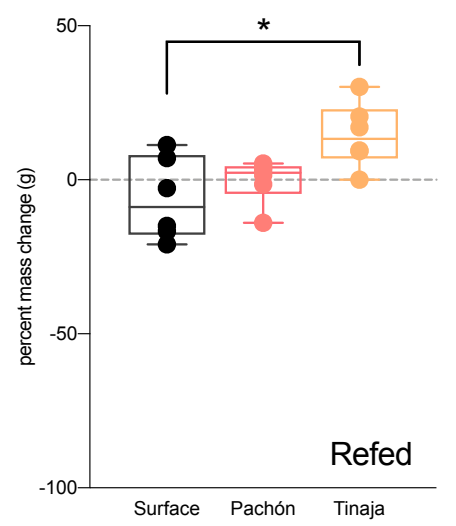

$\mathbf{F}$

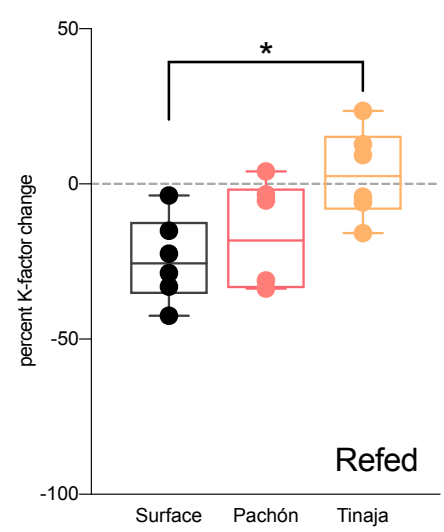

Fig. S2. Percent change of weight and K-factor during fasting regimen. 
bioRxiv preprint doi: https://doi.org/10.1101/2020.10.27.358077; this version posted July 9, 2021. The copyright holder for this preprint (which was not certified by peer review) is the author/funder, who has granted bioRxiv a license to display the preprint in perpetuity. It is made available under aCC-BY-ND 4.0 International license.

A Lipid Data Processing

A

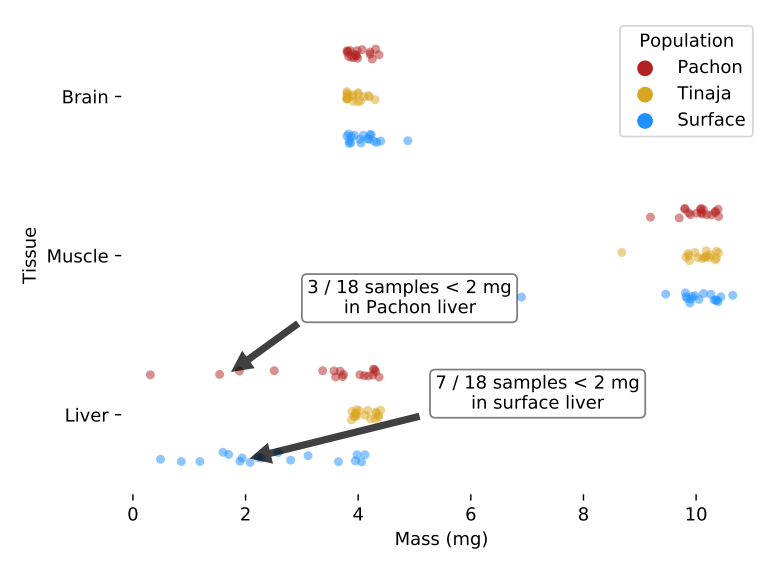

$\mathrm{B}$

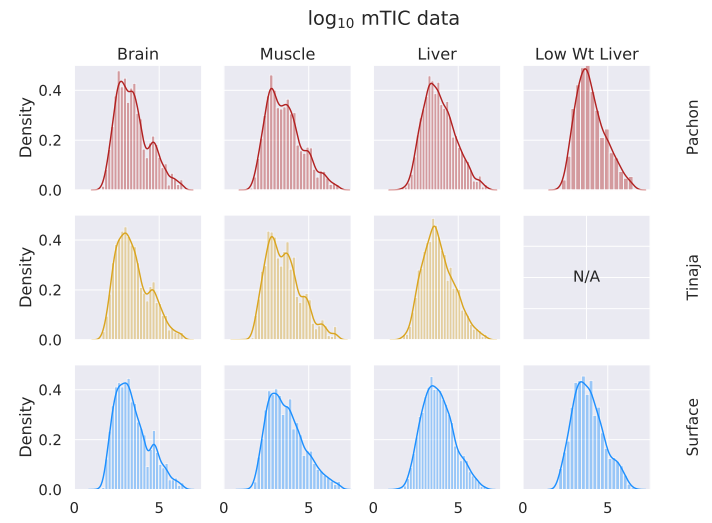

$\mathrm{C}$

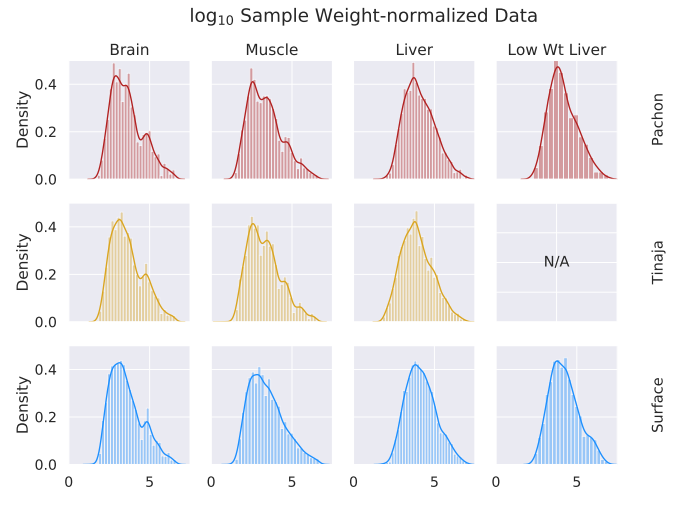

$\mathrm{D}$

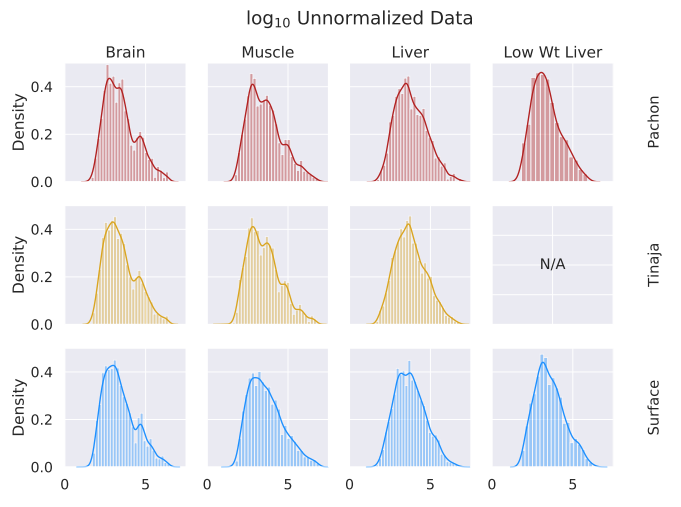

$\mathrm{E}$

\section{Median}

Low Wt. Median

Scheme Tissue Population Samples All

\begin{tabular}{r|c|c|c|c}
\hline mTIC & Liver & Pachon & 3.86 & 3.79 \\
mTIC & Liver & Surface & 3.78 & 3.75 \\
Unnormalized & Liver & Pachon & 3.25 & 3.68 \\
Unnormalized & Liver & Surface & 3.45 & 3.69 \\
Wt. Normalized & Liver & Pachon & 4.03 & 3.97 \\
Wt. Normalized & Liver & Surface & 4.14 & 4.10
\end{tabular}

Fig. S3. Effect of normalization scheme on peak intensity distribution. In comparing the mass of the different tissue samples used in this study (A), we observed that liver samples exhibited more variability than muscle or brain. In particular, some liver samples of Pachón and surface fish had a mass of less than $2 \mathrm{mg}$. In order to adjust for this effect, we compared three different normalization schemes: mTIC normalization (B), wherein each peak is normalized to the sum total of all identified peaks in a given sample (see Methods), (C) sample weight-based normalization, where each peak is normalized according to the physical weight of its sample, and (D) unnormalized peaks. By examining the change in median peak intensity for identified compounds in each method (E), we found that MTIC normalization exhibits slightly better correction for low-weight $(<2 \mathrm{mg})$ median peak intensities compared to weightbased normalization and both are superior to unnormalized data. We therefore employed mTIC normalization for the remainder of the analysis. 

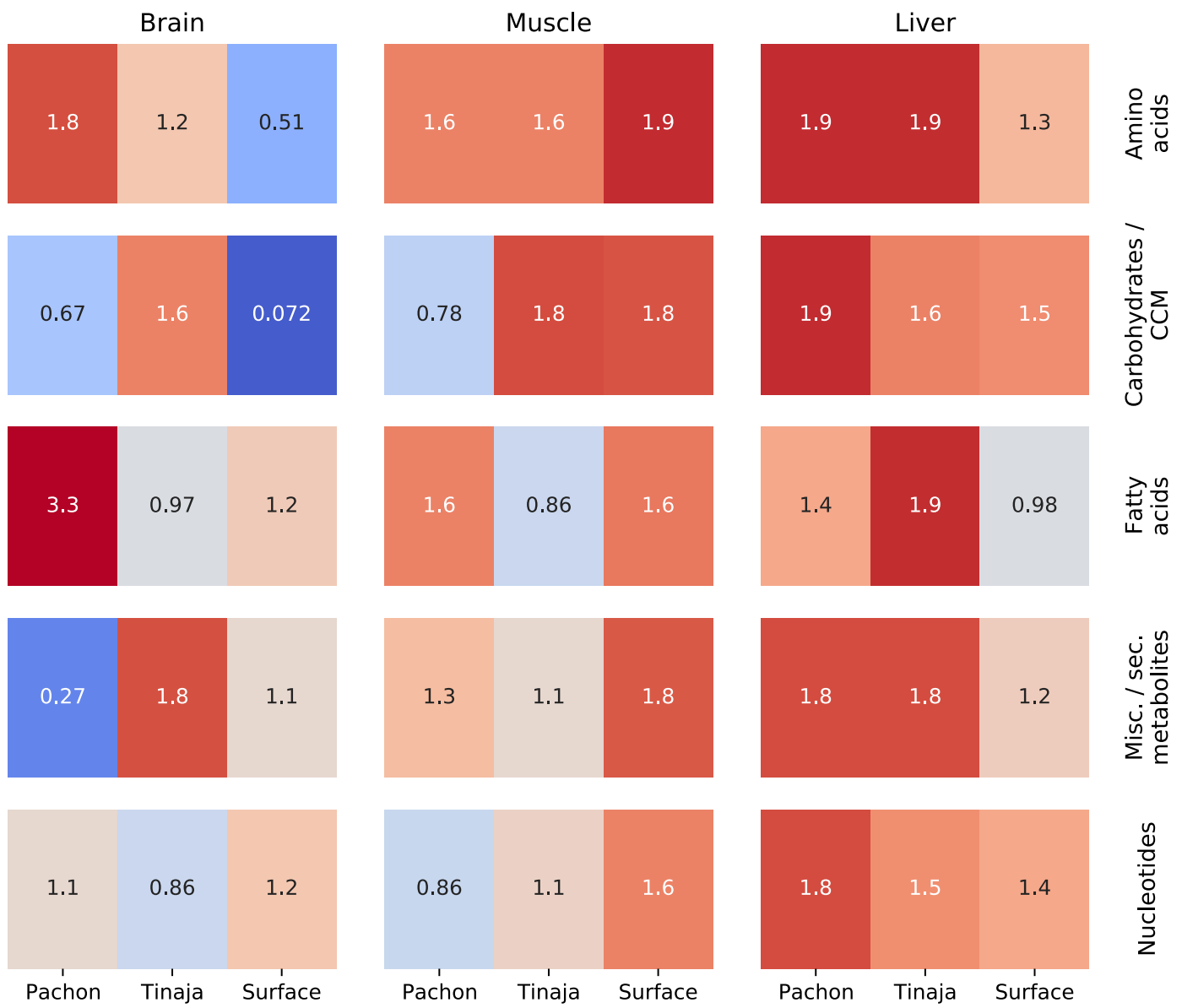

Fig. S4. Classifier performance shows which categories of primary metabolites are most salient in the starvation response. This figure shows $-\log _{10} p$ values of an O-PLS / PLS classifier trained to discriminate 30-day fasted vs refed samples for different tissues, populations, and categories. O-PLS is used to produce de-noised data, which was then fitted to a single-component PLS model. The figure shows $-\log _{10} p$ values for each classifier trained on different subsets of the data corresponding to different categories of primary metabolites. Red indicates the most robust classifiers and blue indicates the least robust. P-values were obtained by randomly permuting the feeding state indices for 2000 iterations and computing the two-tailed survival function of a normal distribution fitted to the $\mathrm{DQ}^{2}$ values of the resulting permuted samples. P-values were adjusted for FDR using the $\mathrm{BH}$ method. 
bioRxiv preprint doi: https://doi.org/10.1101/2020.10.27.358077; this version posted July 9, 2021. The copyright holder for this preprint (which was not certified by peer review) is the author/funder, who has granted bioRxiv a license to display the preprint in perpetuity. It is made available under aCC-BY-ND 4.0 International license.

A Lipid Data Processing
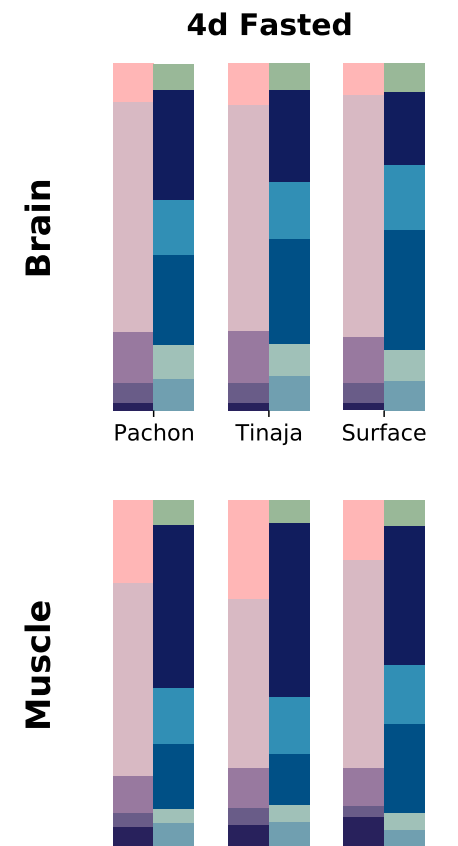

Pachon

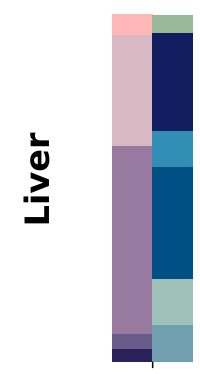

Pachon

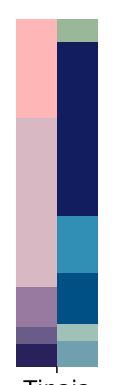

Tinaja

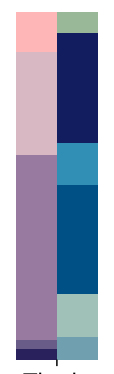

Tinaja
Surface

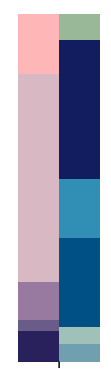

Surface

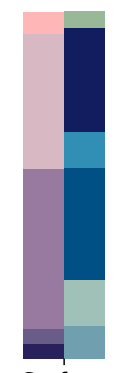

Surface 30d Fasted

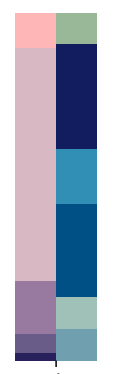

Pachon

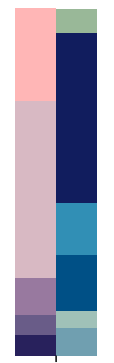

Pachon

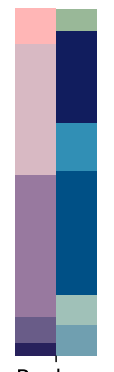

Pachon

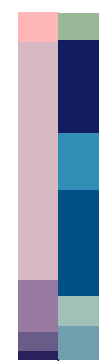

Tinaja

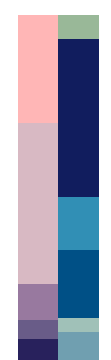

Tinaja

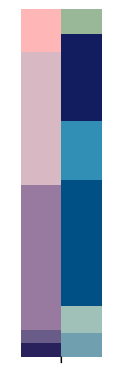

Tinaja

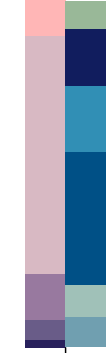

Surface

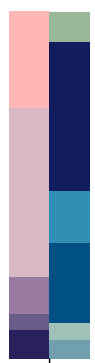

Surface

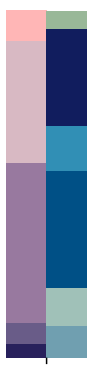

Surface

\section{Refed}

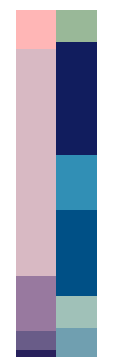

Pachon
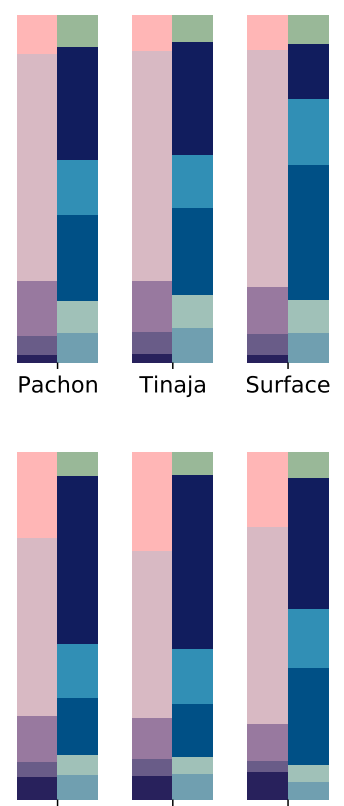

Pachon
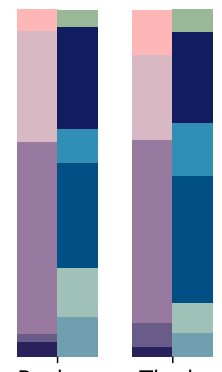

Pachon Tinaja
Primary metabolites

- Nucleotides

Primary fatty acids

Carbohydrates /

CCM

Amino

acids

Misc. / sec.

metabolites

\section{Lipids}

\section{Ceramides} Fatty Acids / Conjugates

- Glycerophosphocholines

Glycerophospho-

ethanolamines

Triradylglycerols

Other lipids

Fig. S5. Relative composition for primary metabolites and lipid classes shows metabolome profile for different tissues, populations, and feeding states. To determine overall composition of the metabolome for different experimental groups, category information for primary metabolites was computed based on five main categories (Methods). Lipid data consists of the five most abundant lipid categories: ceramides, fatty acids and conjugates, glycerophosphocholines, and glycerophosphoethanolamines. Some metabolites, such as palmitate, were detected in both primary and lipid data. The peak intensities for all detected metabolites in these respective categories were summed across all replicates and across positive and negative modes to obtain total peak intensities for each population / condition combination. Total peak intensities were then plotted as a fraction of total peak intensity for all identified metabolites within a given experimental group. 
bioRxiv preprint doi: https://doi.org/10.1101/2020 10.27.358077; this version posted July 9 , 2021. The copyright holder for this preprint (which was not certified by peer review) is the author/funder, who has granted bioRxiv a license to display the preprint in perpetuity. It is made available under aCC-BY-ND 4.0 International license.
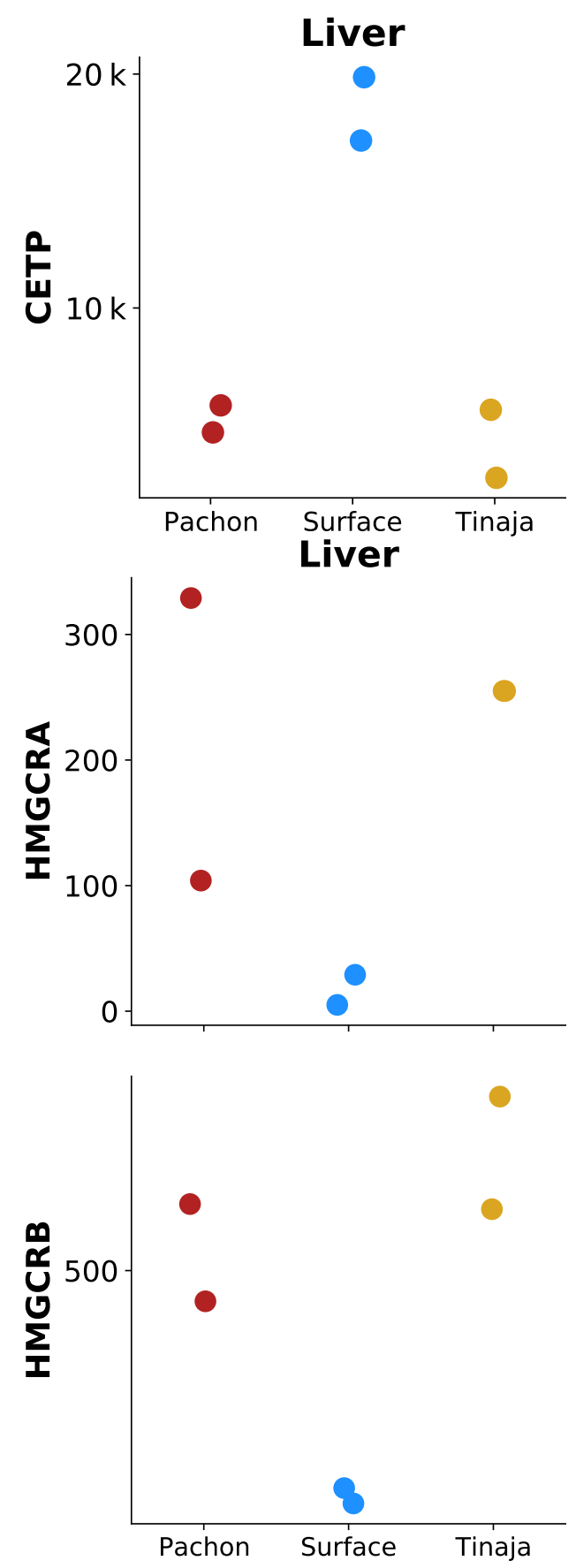

Fig. S6. CETP, but not HMG-CoA reductase homologs, show identical or reduced expression in surface fish. Previously published RNA-Seq data (33) of CETP (ENSAMXG00000003656) and HMGCRA/B (ENSAMXG00000007971 / ENSAMXG00000016175) in cave / surface A. mexicanus. Values on the y-axis are gene expression data in terms of counts measured in liver tissue under normal feeding conditions. 
bioRxiv preprint doi: https://doi.org/10.1101/2020.10.27.358077; this version posted July 9, 2021. The copyright holder for this preprint (which was not certified by peer review) is the author/funder, who has granted bioRxiv a license to display the preprint in perpetuity. It is made available under aCC-BY-ND 4.0 International license.

A Lipid Data Processing

A

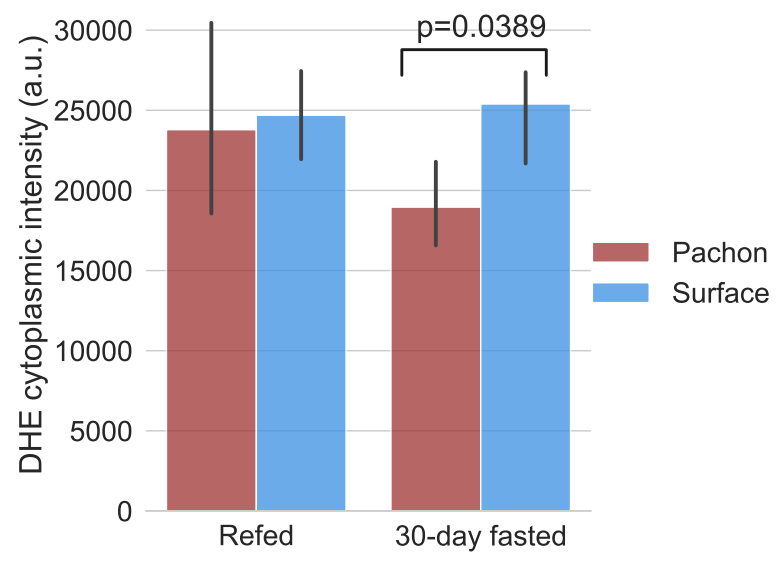

$\mathrm{C}$

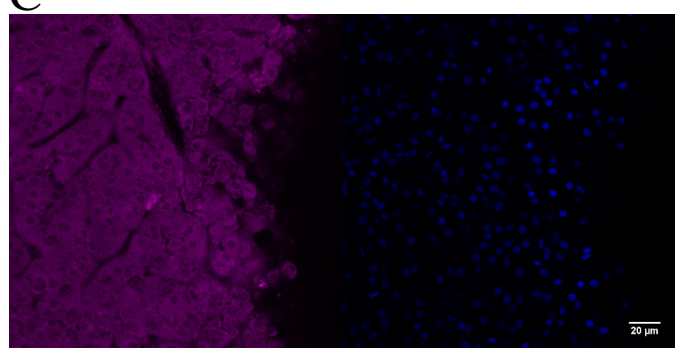

$\mathrm{E}$

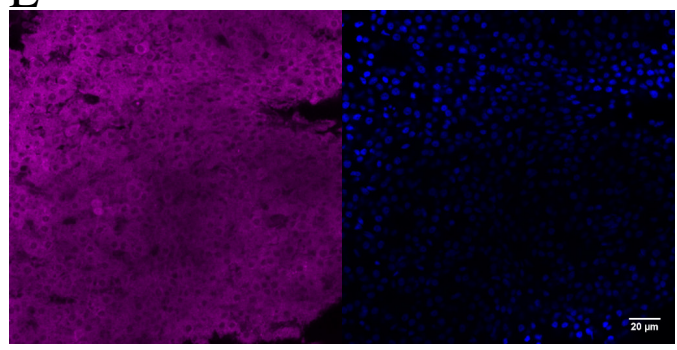

B

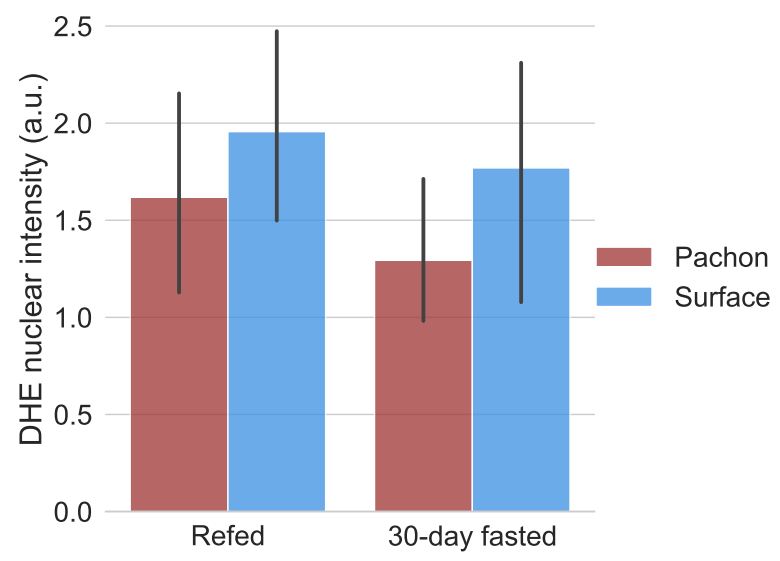

$\mathrm{D}$

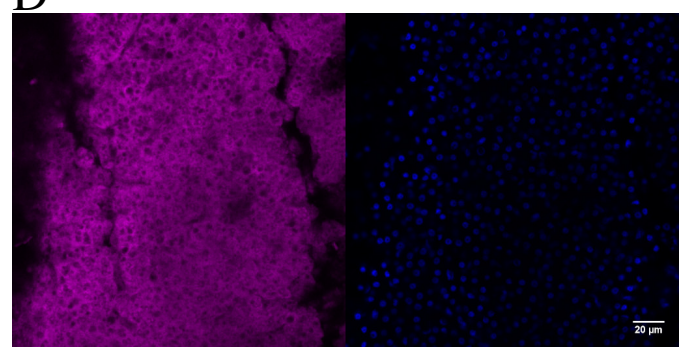

$\mathrm{F}$

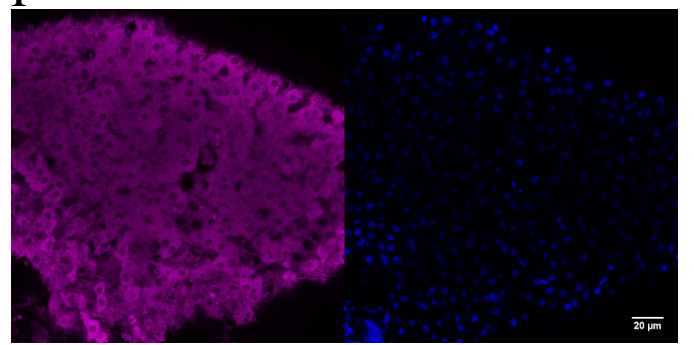

Fig. S7. ROS levels in refed / fasted liver via DHE staining. Pachón cavefish and surface fish were fasted for 30 days and livers were removed and stained with dihydroethidium (DHE), a blue-fluorescent dye that fluoresces red when oxidized and serves as an indicator of the presence of superoxide radicals. While we did not detect a significant difference in baseline staining in fish refed after 4 days of fasting, cytoplasmic ROS in Pachón was significantly (Kruskal-Wallis test) decreased in Pachón with respect to surface as measured by DHE staining (A). Nuclear differences were not detected among any groups (B). (C-F): Arbitrarily selected images of DHE (left) and DAPI (right) staining for Pachón with 4-day fasting followed by refeeding (C), surface with 4-day fasting followed by refeeding (D), Pachón with 30-day fasting (E), and surface with 30-day fasting (F). All images are available at https://stowersinstitute-my.sharepoint.com/:u:/g/personal/jm2432_stowers_org/ ETQkzlnbUN1CmXDdKcEUI48BdfHa8Ur6DAl3fdE1YGs_Xw?e=BEhNhc. 
bioRxiv preprint doi: https://doi.org/10.1101/2020.10.27.358077; this version posted July 9,2021 . The copyright holder for this preprint (which was not certified by peer review) is the author/funder, who has granted bioRxiv a license to display the preprint in perpetuity. It is made available under aCC-BY-ND 4.0 International license.

Treat- Initial Final Initial Final Initial Final Weight K-factor Pop. ment Sample mass (g) mass (g) SL (cm) SL (cm) K-factor K-factor Chg. (\%) Chg. (\%)

\begin{tabular}{|c|c|c|c|c|c|c|c|c|c|c|}
\hline \multirow{18}{*}{$\begin{array}{l}\stackrel{\mathscr{U}}{\tilde{E}} \\
\stackrel{\Xi}{\Xi}\end{array}$} & $30 \mathrm{~d}$ & S30.1 & 0.47 & 0.35 & 2.8 & 2.9 & 2.14 & 1.44 & -34.3 & -49.2 \\
\hline & $30 \mathrm{~d}$ & S 30.2 & 1.00 & 0.84 & 3.4 & 3.6 & 2.54 & 1.80 & -19.0 & -41.3 \\
\hline & $30 \mathrm{~d}$ & S30.3 & 0.89 & 0.48 & 3.4 & 3.4 & 2.26 & 1.22 & -85.4 & -85.4 \\
\hline & $30 \mathrm{~d}$ & S30.4 & 0.86 & 0.64 & 3.2 & 3.3 & 2.62 & 1.78 & -34.4 & -47.4 \\
\hline & $30 \mathrm{~d}$ & S30.5 & 0.83 & 0.66 & 3.3 & 3.3 & 2.31 & 1.84 & -25.8 & -25.8 \\
\hline & $30 \mathrm{~d}$ & S30.6 & 1.72 & 1.29 & 4.2 & 4.2 & 2.32 & 1.74 & -33.3 & -33.3 \\
\hline & $4 d$ & S4.1 & 0.43 & 0.43 & 2.7 & 2.8 & 2.18 & 1.96 & 0.0 & -11.5 \\
\hline & $4 d$ & S4.2 & 1.01 & 0.88 & 3.4 & 3.9 & 2.57 & 1.48 & -14.8 & -73.2 \\
\hline & $4 d$ & S4.3 & 0.60 & 0.53 & 3.1 & 3.2 & 2.01 & 1.62 & -13.2 & -24.5 \\
\hline & $4 d$ & S4.4 & 0.86 & 0.8 & 3.3 & 3.7 & 2.39 & 1.58 & -7.5 & -51.5 \\
\hline & $4 d$ & S4.5 & 1.71 & 1.9 & 4.1 & 4.5 & 2.48 & 2.18 & 14.1 & -13.6 \\
\hline & $4 d$ & S4.6 & 0.51 & 0.48 & 2.9 & 3.0 & 2.09 & 1.78 & -6.3 & -17.6 \\
\hline & Ref & SR.1 & 0.46 & 0.4 & 2.7 & 2.9 & 2.34 & 1.64 & -15.0 & -42.5 \\
\hline & Ref & SR.2 & 0.56 & 0.48 & 3.0 & 3.1 & 2.07 & 1.61 & -16.7 & -28.7 \\
\hline & Ref & SR.3 & 0.40 & 0.43 & 2.7 & 2.8 & 2.03 & 1.96 & 7.0 & -3.7 \\
\hline & Ref & SR.4 & 0.75 & 0.62 & 3.1 & 3.2 & 2.52 & 1.89 & -21.0 & -33.1 \\
\hline & Ref & SR.5 & 0.77 & 0.75 & 3.3 & 3.5 & 2.14 & 1.75 & -2.7 & -22.5 \\
\hline & Ref & SR.6 & 0.86 & 0.97 & 3.3 & 3.6 & 2.39 & 2.08 & 11.3 & -15.1 \\
\hline \multirow{18}{*}{ 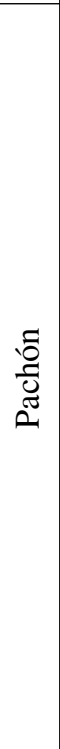 } & $30 \mathrm{~d}$ & P30.1 & 1.03 & 0.96 & 3.7 & 3.7 & 2.03 & 1.90 & -7.3 & -7.3 \\
\hline & $30 \mathrm{~d}$ & P30.2 & 1.12 & 0.83 & 3.6 & 3.5 & 2.40 & 1.94 & -34.9 & -24.0 \\
\hline & $30 \mathrm{~d}$ & P30.3 & 1.17 & 0.99 & 3.6 & 3.5 & 2.51 & 2.31 & -18.2 & -8.6 \\
\hline & $30 \mathrm{~d}$ & P30.4 & 1.29 & 1.06 & 3.7 & 3.8 & 2.55 & 1.93 & -21.7 & -31.8 \\
\hline & $30 \mathrm{~d}$ & P30.5 & 1.02 & 0.81 & 3.5 & 3.5 & 2.38 & 1.89 & -25.9 & -25.9 \\
\hline & $30 \mathrm{~d}$ & P30.6 & 0.73 & 0.55 & 3.3 & 3.4 & 2.03 & 1.40 & -32.7 & -45.2 \\
\hline & $4 d$ & P4.1 & 0.82 & 0.81 & 3.3 & 3.5 & 2.28 & 1.89 & -1.2 & -20.8 \\
\hline & $4 d$ & P4.2 & 1.00 & 1.01 & 3.5 & 3.5 & 2.33 & 2.36 & 1.0 & 1.0 \\
\hline & $4 d$ & P4.3 & 1.34 & 1.53 & 3.8 & 4.1 & 2.44 & 2.22 & 12.4 & -10.0 \\
\hline & $4 d$ & P4.4 & 0.32 & 0.37 & 2.6 & 2.8 & 1.82 & 1.69 & 13.5 & -8.0 \\
\hline & $4 d$ & P4.5 & 1.05 & 1.16 & 3.7 & 3.7 & 2.07 & 2.29 & 9.5 & 9.5 \\
\hline & $4 d$ & P4.6 & 1.09 & 1.12 & 3.6 & 3.7 & 2.24 & 2.21 & 2.7 & -1.4 \\
\hline & Ref & PR.1 & 1.06 & 0.93 & 3.7 & 3.9 & 2.09 & 1.57 & -14.0 & -33.5 \\
\hline & Ref & PR.2 & 0.97 & 1.00 & 3.6 & 3.7 & 2.08 & 1.97 & 3.0 & -5.3 \\
\hline & Ref & PR.3 & 0.89 & 0.94 & 3.4 & 3.5 & 2.26 & 2.19 & 5.3 & -3.3 \\
\hline & Ref & PR.4 & 0.75 & 0.74 & 3.1 & 3.4 & 2.52 & 1.88 & -1.4 & -33.7 \\
\hline & Ref & PR.5 & 1.18 & 1.23 & 3.7 & 3.7 & 2.33 & 2.43 & 4.1 & 4.1 \\
\hline & Ref & PR.6 & 0.66 & 0.67 & 3.0 & 3.3 & 2.44 & 1.86 & 1.5 & -31.1 \\
\hline \multirow{18}{*}{ 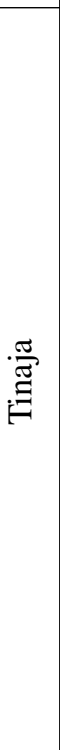 } & $30 \mathrm{~d}$ & T30.1 & 0.99 & 0.70 & 3.5 & 3.7 & 2.31 & 1.38 & -41.4 & -67.1 \\
\hline & $30 \mathrm{~d}$ & T30.2 & 1.37 & 1.12 & 3.9 & 4.0 & 2.31 & 1.75 & -22.3 & -32.0 \\
\hline & $30 \mathrm{~d}$ & T30.3 & 1.64 & 1.37 & 4.0 & 4.3 & 2.56 & 1.72 & -19.7 & -48.7 \\
\hline & $30 \mathrm{~d}$ & T30.4 & 1.08 & 0.93 & 3.7 & 3.7 & 2.13 & 1.84 & -16.1 & -16.1 \\
\hline & $30 \mathrm{~d}$ & T30.5 & 0.94 & 1.02 & 3.5 & 3.5 & 2.19 & 2.38 & 7.8 & 7.8 \\
\hline & $30 \mathrm{~d}$ & T30.6 & 0.72 & 0.75 & 3.5 & 3.5 & 1.68 & 1.75 & 4.0 & 4.0 \\
\hline & $4 d$ & $\mathrm{~T} 4.1$ & 1.60 & 1.82 & 4.1 & 4.3 & 2.32 & 2.29 & 12.1 & -1.4 \\
\hline & $4 d$ & $\mathrm{~T} 4.2$ & 0.86 & 0.84 & 3.2 & 3.4 & 2.62 & 2.14 & -2.4 & -22.8 \\
\hline & $4 d$ & $\mathrm{~T} 4.3$ & 0.96 & 0.78 & 3.4 & 3.6 & 2.44 & 1.67 & -23.1 & -46.1 \\
\hline & $4 d$ & T4.4 & 1.22 & 1.10 & 3.7 & 3.8 & 2.41 & 2.00 & -10.9 & -20.1 \\
\hline & $4 d$ & T4.5 & 1.56 & 1.68 & 4.1 & 4.3 & 2.26 & 2.11 & 7.1 & -7.1 \\
\hline & $4 d$ & T4.6 & 1.15 & 1.01 & 3.6 & 3.7 & 2.46 & 1.99 & -13.9 & -23.6 \\
\hline & Ref & TR.1 & 1.23 & 1.36 & 3.7 & 3.9 & 2.43 & 2.29 & 9.6 & -5.9 \\
\hline & Ref & TR.2 & 0.74 & 1.06 & 3.3 & 3.4 & 2.06 & 2.70 & 30.2 & 23.6 \\
\hline & Ref & TR.3 & 1.31 & 1.58 & 3.8 & 4.1 & 2.39 & 2.29 & 17.1 & -4.1 \\
\hline & Ref & TR.4 & 0.66 & 0.83 & 3.2 & 3.3 & 2.01 & 2.31 & 20.5 & 12.8 \\
\hline & Ref & TR.5 & 0.98 & 1.08 & 3.6 & 3.6 & 2.10 & 2.31 & 9.3 & 9.3 \\
\hline & Ref & TR.6 & 1.48 & 1.48 & 4.0 & 4.2 & 2.31 & 2.00 & 0.0 & -15.8 \\
\hline
\end{tabular}

Table S1. Weight and K-factor measurements of all samples. 
bioRxiv preprint doi: https://doi.org/10.1101/2020.10.27.358077; this version posted July 9, 2021. The copyright holder for this preprint (which was not certified by peer review) is the author/funder, who has granted bioRxiv a license to display the preprint in perpetuity. It is made available under aCC-BY-ND 4.0 International license.

A Lipid Data Processing

Table S2. An Excel file containing the results of the O-PLS / GLM feature identification pipeline for primary metabolites divided into categories. Columns represent different population and feeding condition combinations. https://drive.google.com/file/d/ 1ax0I3_BWO5-MLwWivA5iyVTI_wU4 jCBs/view?usp=sharing

\begin{tabular}{|c|c|c|c|c|c|c|c|c|c|c|c|c|c|c|c|c|c|c|c|c|c|c|c|c|c|c|c|}
\hline & \multicolumn{9}{|c|}{ Pachon vs. Surface } & \multicolumn{9}{|c|}{ Tinaja vs. Surface } & \multicolumn{9}{|c|}{ Pachon vs. Tinaja } \\
\hline Fatty Acyls & & & & & & & & - & & & & & & & & & - & - & & & & & & & & & + \\
\hline Glycerophospholipids & - & - & - & & - & & & & & & & - & & & - & & & & & & & & & & & & \\
\hline Sphingolipids & & & & + & + & + & & & & & & & + & + & + & - & - & - & & & & & & & + & + & + \\
\hline Sterol Lipids & & - & - & & & & & & & & & - & & & & & & - & & & & & & & & & \\
\hline
\end{tabular}

Table S3. Interpopulation Differences in Abundance of Lipid Categories Peak intensities for all lipids in a given category (determined from the LipidMaps "CATEGORY" attribute) were summed to yield a total intensity for each category which is either significantly (at the $p<0.05$ level) up- (+) or down-regulated (-) in a given cave population with respect to surface (Pachón versus surface and Tinaja versus surface, top row) or the Pachón cave population with respect to the Tinaja cave population (last comparison, top row). The sample set for each tissue / feeding state combination consists of six individuals from each population as shown in Fig 2. P-values were obtained from the OPLS / GLM approach described in Methods. Coloring $(\square$ indicates a class that agrees in significance and directionality between both cave populations and is thus may be related to cave adaptation. Columns corresponding to muscle are highlighted to help distinguish the different tissues. Compare Table S4 based on the "MAIN_CLASS" attribute.

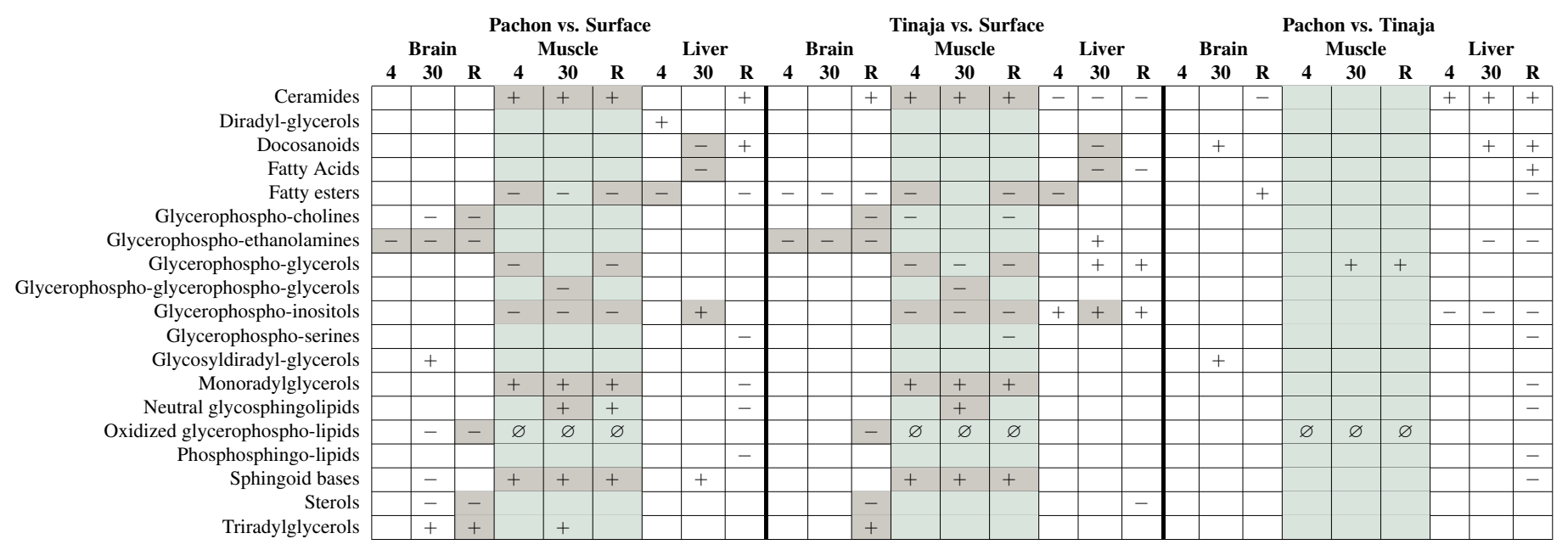

Table S4. Interpopulation Differences in Abundance of Lipid Classes Significant increased $(+)$ or decreased (-) lipid classes based on summed peak intensities for every lipid species belonging to a given LipidMaps "MAIN_CLASS" label. $\varnothing$ denotes classes which were not detected in a given sample set. LipidMaps also possesses a "CATEGORY" attribute that provides a more coarse-grained classification of lipid species, which is used as a basis for a similar analysis shown in Table S3.

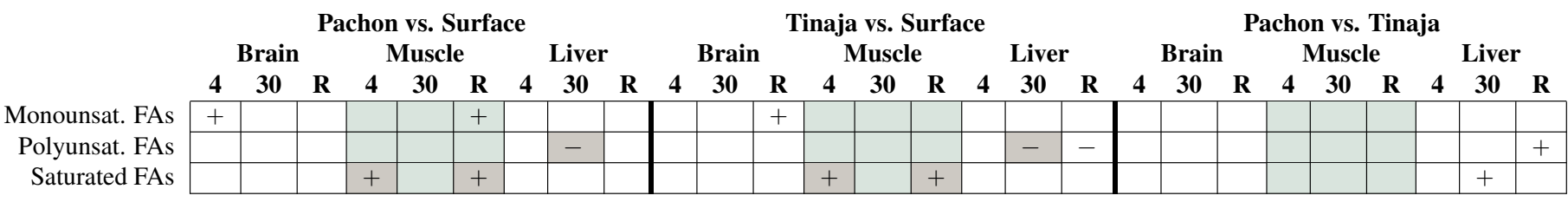

Table S5. Intra-population Differences in Fatty Acid Saturation For lipids corresponding to free fatty acids, sauturation was calculated based on the presence of double bonds in LipidMaps structural data and used to classify each LMID as either saturated, monounsaturated, or polyunsaturated. Significance values were again calculated using an OPLS / Bayesian GLM workflow. Coloring and markings as before. 
bioRxiv preprint doi: https://doi.org/10.1101/2020.10.27.358077; this version posted July 9, 2021. The copyright holder for this preprint (which was not certified by peer review) is the author/funder, who has granted bioRxiv a license to display the preprint in perpetuity. It is made available under aCC-BY-ND 4.0 International license.

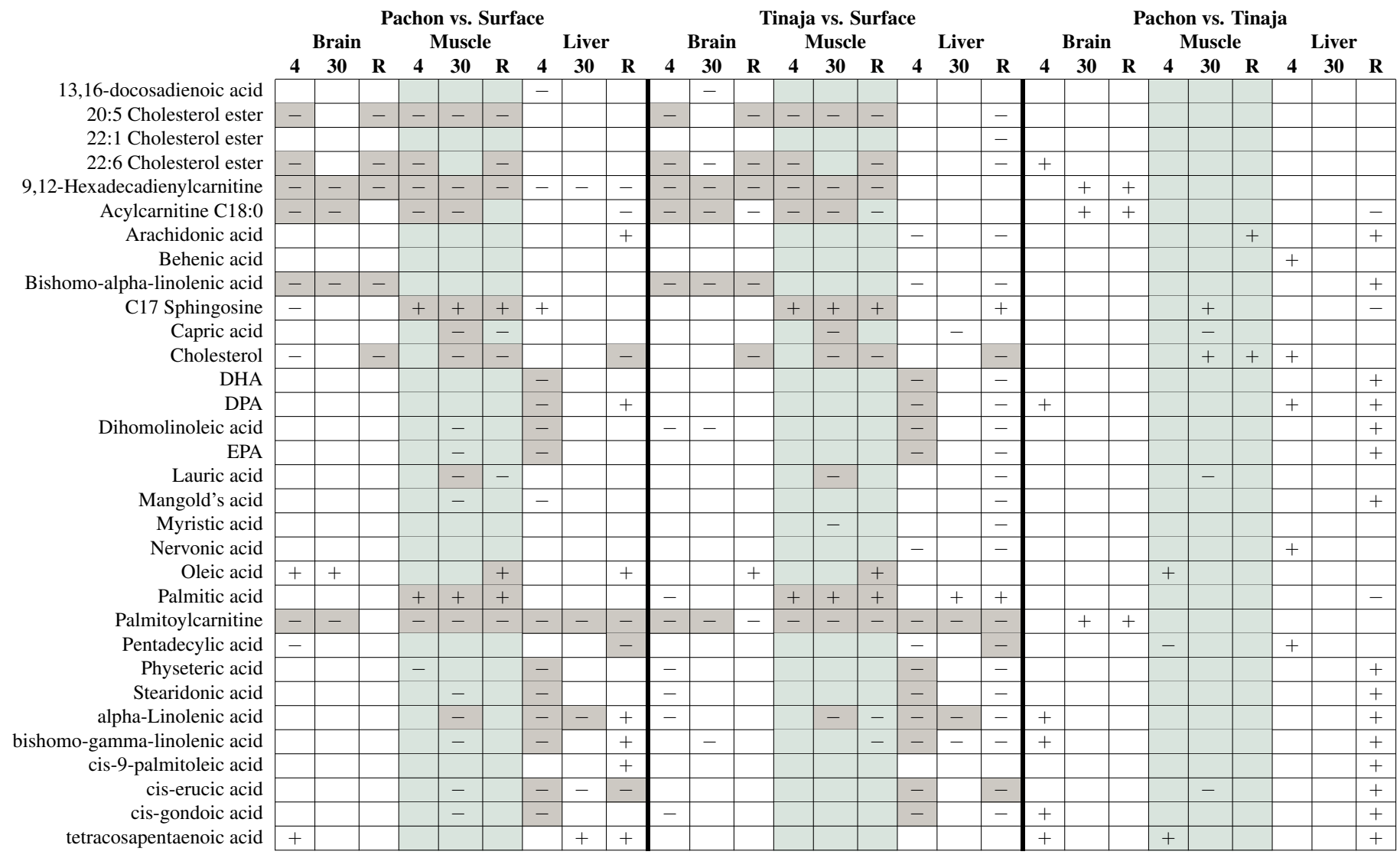

Table S6. Common lipids Lipids with common names were selected from the set of 447 identified lipids and analyzed using the O-PLS / GLM scheme. Many lipids exhibit a strongly conserved pattern between Pachón and Tinaja, and these are highlighted in gray. Of note, omega-3 fatty acids appear to exhibit lower abundance in the 4-day fasted state in liver. Some metabolites, such as palmitate and oleic acid, overlap with primary metabolomics data. 


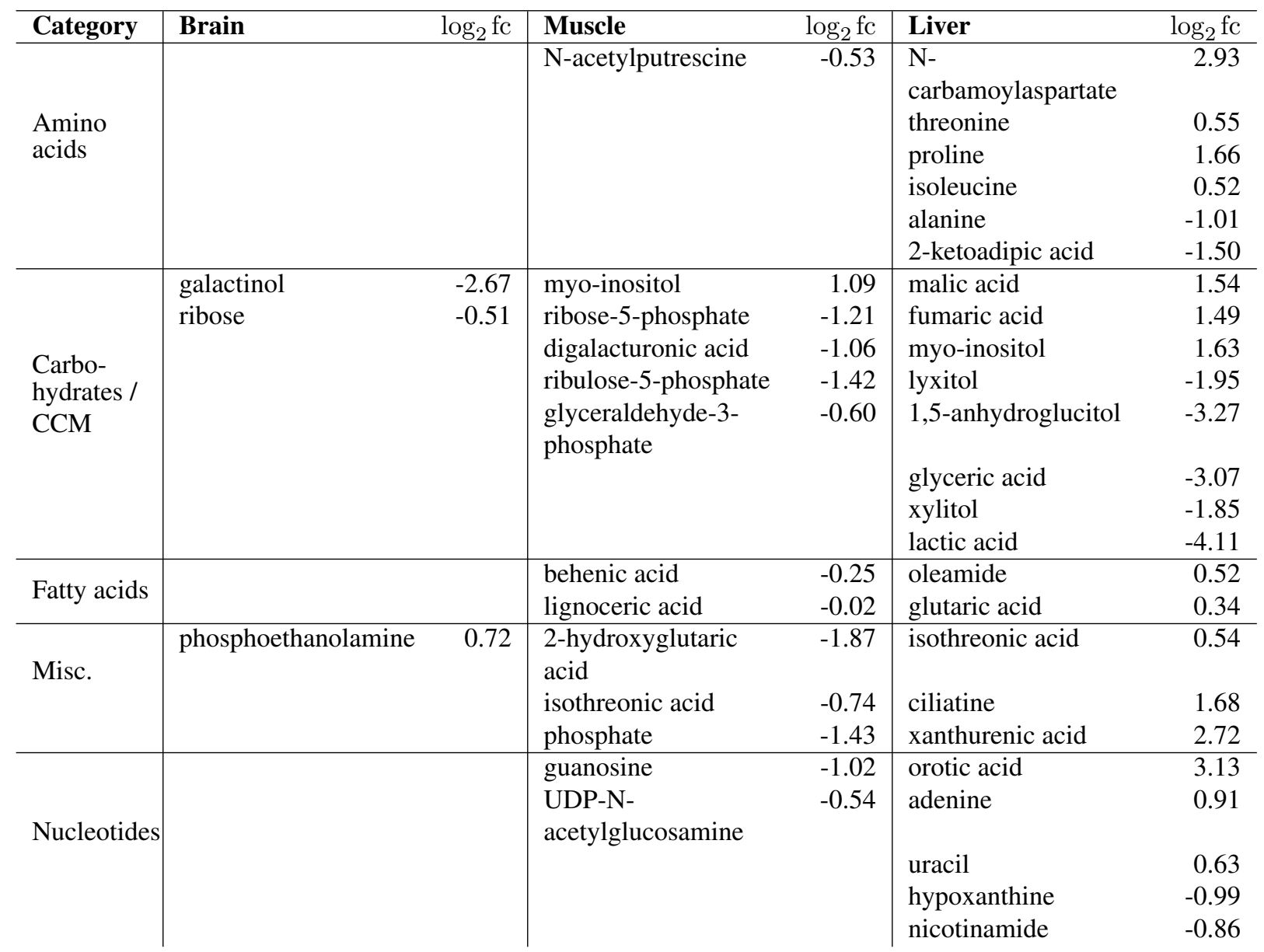

Table S7. Adaptive Response in 30-day Fasting. Differentially significant metabolites in 30-day fasted states which are similar in both cave populations. This table shows $\mathrm{p}-\mathrm{values}$ associated with a logistic regression model using OPLS-filtered z-scores for 30-day tasted and refed fish as input. To identify metabolites conserved between both cave populations, we implemented the test $0.5 \cdot(\mathrm{PS}-\mathrm{PR}+\mathrm{TS}-\mathrm{TR})-(\mathrm{SS}-\mathrm{SR})$ where PS refers to Pachón 30-day fasted, PR refers to Pachón refed, etc. Tables S8 and S9 show conserved metabolites in cave populations versus surface for 4-day fasted versus refed and 30-day fasted versus 4-day fasted respectively. $\log _{2}$ fc values are also based on this formula. Outliers were excluded for this analysis (Fig ??). 
bioRxiv preprint doi: https://doi.org/10.1101/2020.10.27.358077; this version posted July 9, 2021. The copyright holder for this preprint (which was not certified by peer review) is the author/funder, who has granted bioRxiv a license to display the preprint in perpetuity. It is made available under aCC-BY-ND 4.0 International license.

\begin{tabular}{|c|c|c|c|c|c|c|}
\hline Category & Brain & $\log _{2} \mathrm{fc}$ & Muscle & $\log _{2} \mathrm{fc}$ & Liver & $\log _{2} f \mathrm{c}$ \\
\hline \multirow{4}{*}{$\begin{array}{l}\text { Amino } \\
\text { acids }\end{array}$} & $\mathrm{N}$-acetylaspartic acid & -0.23 & shikimic acid & -0.75 & tryptophan & 0.69 \\
\hline & & & glutamic acid & -0.73 & proline & 1.01 \\
\hline & & & & & & 3.03 \\
\hline & & & & & carbamoylaspartate & \\
\hline \multirow{5}{*}{$\begin{array}{l}\text { Carbo- } \\
\text { hydrates / } \\
\text { CCM }\end{array}$} & sucrose & 1.49 & ribose-5-phosphate & -2.01 & & \\
\hline & glucoheptulose & -1.50 & & & & \\
\hline & threonic acid & -0.50 & & & & \\
\hline & maltose & -1.15 & & & & \\
\hline & maltotriose & -1.40 & & & & \\
\hline Fatty acids & $\begin{array}{l}\text { 2-hydroxybutanoic } \\
\text { acid }\end{array}$ & -2.35 & & & oleic acid & -1.63 \\
\hline \multirow{6}{*}{ Misc. } & 2-aminobutyric acid & -0.54 & phosphoethanolamine & -1.08 & isothreonic acid & 0.45 \\
\hline & & & 4-hydroxybenzoate & -0.55 & & \\
\hline & & & 2-hydroxyglutaric & -2.67 & & \\
\hline & & & acid & & & \\
\hline & & & maleimide & -0.56 & & \\
\hline & & & ciliatine & -0.81 & & \\
\hline \multirow{5}{*}{ Nucleotides } & & & $\begin{array}{l}\text { UDP-N- } \\
\text { acetylglucosamine }\end{array}$ & -0.82 & orotic acid & 3.08 \\
\hline & & & cytosine & -0.69 & inosine & -0.34 \\
\hline & & & uridine & -1.12 & & \\
\hline & & & guanosine & -1.39 & & \\
\hline & & & uracil & -1.15 & & \\
\hline
\end{tabular}

Table S8. Adaptive Response in 4-day Fasting. Differentially significant metabolites in 4-day fasted states which are similar in both cave populations. Cf. Table S7 with the difference that this table compares 4-day fasted versus refed conditions. Thus, metabolites displayed as upregulated in this table are differentially upregulated in 4-day fasted cave fish versus refed cave fish using surface fish as a baseline for comparison. Outliers are not included in this analysis.

\begin{tabular}{|c|c|c|c|c|c|c|}
\hline Category & Brain & $\log _{2} f \mathrm{c}$ & Muscle & $\log _{2} \mathrm{fc}$ & Liver & $\log _{2} \mathrm{fc}$ \\
\hline \multirow{4}{*}{$\begin{array}{l}\text { Amino } \\
\text { acids }\end{array}$} & & & & & oxoproline & 0.79 \\
\hline & & & & & glutamic acid & 1.45 \\
\hline & & & & & threonine & 0.67 \\
\hline & & & & & alanine & -1.13 \\
\hline \multirow{6}{*}{$\begin{array}{l}\text { Carbo- } \\
\text { hydrates / } \\
\text { CCM }\end{array}$} & maltotriose & 3.13 & myo-inositol & 1.26 & myo-inositol & 1.08 \\
\hline & threonic acid & 0.50 & digalacturonic acid & -1.49 & glucose & 0.40 \\
\hline & sophorose & -1.36 & & & raffinose & -1.89 \\
\hline & isomaltose & -1.83 & & & maltotriose & -1.07 \\
\hline & & & & & xylitol & -1.50 \\
\hline & & & & & 1-kestose & -1.82 \\
\hline \multirow{2}{*}{ Fatty acids } & stearic acid & -0.25 & palmitic acid & -0.24 & stearic acid & -0.05 \\
\hline & & & & & palmitic acid & 0.06 \\
\hline \multirow{3}{*}{ Misc. } & & & phosphate & -2.71 & 2-aminobutyric acid & 1.54 \\
\hline & & & glycolic acid & -0.99 & ciliatine & 1.37 \\
\hline & & & pyrophosphate & -1.28 & xanthurenic acid & 1.65 \\
\hline \multirow{2}{*}{ Nucleotides } & adenosine & 1.87 & xanthine & 0.76 & & \\
\hline & & & inosine & 0.92 & & \\
\hline
\end{tabular}

Table S9. Adaptive Response in 30- vs 4-day Fasting. Differentially significant metabolites in 30-day vs 4-day fasting which are similar between both cave populations. Cf. Table S7 with the differences that this table compares 30-day fasted versus 4-day fasted conditions. Thus, metabolites displayed as upregulated in this table are differentially upregulated in 30-day fasted cave fish versus 4-day fasted cave fish using surface fish as a baseline for comparison. Outliers are not included in this analysis.

Table S10. All caudal vein images using Van Gieson staining. https://stowersinstitute-my. sharepoint.com/: u:/g/ personal/jm2432_stowers_org/ESotYEumMNZGt 91PEM-TLIUBUy8SqS0bxwTyuyPOXsFyGg?e=YESdnP 
bioRxiv preprint doi: https://doi.org/10.1101/2020.10.27.358077; this version posted July 9, 2021. The copyright holder for this preprint (which was not certified by peer review) is the author/funder, who has granted bioRxiv a license to display the preprint in perpetuity. It is made available under aCC-BY-ND 4.0 International license.

A Lipid Data Processing

\begin{tabular}{|l|l|l|l|l|}
\hline Popuation & Sample & Weight $(\mathrm{g})$ & Length $(\mathrm{mm})$ & Sex \\
\hline Surface & 1 & 21.9 & 86 & $\mathrm{~F}$ \\
Surface & 2 & 15.4 & 84.39 & $\mathrm{~F}$ \\
Surface & 3 & 9.4 & 73.2 & M \\
Pachon & 1 & 12.22 & 77.96 & M \\
Pachon & 2 & 17.42 & 86.35 & M \\
Pachon & 3 & 14.98 & 83.72 & M \\
\hline
\end{tabular}

Table S11. Info and measurements for fish used in HDL/LDL/VLDL assay (Fig 8. Weight (in grams), length (in millimeters), and sex for each fish used in the lipoprotein assay (Fig 8.

\begin{tabular}{|l|l|l|l|l|l|l|l|}
\hline & & \multicolumn{3}{|c|}{ TR1 } & \multicolumn{3}{c|}{ TR2 } \\
\hline Sample & Spike $(\mu \mathrm{l})$ & Std & HDL & LDL/VLDL & Std & HDL & LDL/VLDL \\
\hline Pachon 1 & 0 & 0.0436 & 0.3187 & 0.22 & 0.0456 & 0.3024 & 0.2275 \\
Surface 1 & 1 & 0.3468 & 0.639 & 0.5749 & 0.3425 & 0.638 & 0.5654 \\
Pachon 2 & 2 & 0.6086 & 0.2225 & 0.9198 & 0.6049 & 0.2784 & 0.884 \\
Surface 2 & 3 & 0.8506 & 0.6009 & 0.4745 & 0.8641 & 0.5986 & 0.4498 \\
Pachon 3 & 4 & 1.0737 & 0.3259 & 0.8434 & 1.0977 & 0.3346 & 0.8046 \\
Surface 3 & 5 & 1.2895 & 0.5852 & 0.4625 & 1.3258 & 0.5632 & 0.474 \\
\hline
\end{tabular}

Table S12. Brightness readings for cholesterol assay. Source data for Fig 8. Three fish each from Pachòn and surface populations resp. were euthanized according to protocol and blood was collected from the caudal vein and blood samples were prepared as described in Methods. Blood samples were 50-fold diluted. Cholesterol standards provided with the Sigma-Aldrich HDL and LDL/VLDL quantitation kit were spiked into separate wells at the concentrations shown above. Readings were taken on a Spectramax id3 plate reader for two technical replicates of each sample and raw readings are reported above. 